\title{
THE UNIQUENESS AND STABILITY \\ OF THE SOLUTION OF THE RIEMANN PROBLEM OF A SYSTEM OF CONSERVATION LAWS OF MIXED TYPE
}

\author{
HAITAO FAN
}

\begin{abstract}
We establish the uniqueness and stability of the similarity solution of the Riemann problem for a $2 \times 2$ system of conservation laws of mixed type, with initial data separated by the elliptic region, which satisfies the viscositycapillarity travelling wave admissibility criterion.
\end{abstract}

\section{INTRODUCTION}

The isothermal evolution of one-dimensional continuous compressible media in the absence of body forces can be described in Lagrangian coordinates by the quasilinear system of conservation laws

$$
\begin{aligned}
u_{t}+p(w)_{x} & =0, \\
w_{t}-u_{x} & =0, \quad x \in \mathbb{R}, t>0,
\end{aligned}
$$

where $p(w)$ is the pressure. Typically, for instance in ideal gases, $p^{\prime}(w)<0$, so that the system $(1.1 \mathrm{a}, \mathrm{b})$ is hyperbolic. For some other material models, for example the van der Waals gas or elastic/plastic rods, $p^{\prime}(w)$ may be positive on some range of $w$, as depicted in Figure 1. More precisely, we assume

$$
\begin{gathered}
p(w) \in C^{1}(\mathbb{R}) \text { and } p^{\prime}(w)<0 \text { for } w \notin[\alpha, \beta], \\
p^{\prime}(w)>0 \text { for } w \in(\alpha, \beta) .
\end{gathered}
$$

With this kind of function $p(w)$, the system $(1.1 \mathrm{a}, \mathbf{b})$ is of hyperbolic-elliptic mixed type.

In this paper, we shall continue the program carried out in $[20,7]$ to study the system $(1.1 \mathrm{a}, \mathrm{b}, \mathrm{c})$ with the following Riemann initial values

$$
\begin{gathered}
(u(x, 0), w(x, 0))= \begin{cases}\left(u_{-}, w_{-}\right) & \text {for } x<0, \\
\left(u_{+}, w_{+}\right) & \text {for } x>0,\end{cases} \\
w_{-}<\alpha<\beta<w_{+} .
\end{gathered}
$$

The system (1.1) generally admits many solutions but not every one of them is physically relevant. This raises the issue of the admissibility of these solutions.

Received by the editors August 30, 1990.

1980 Mathematics Subject Classification (1985 Revision). Primary 35L65; Secondary 35L67, 76L05.

Key words and phrases. Viscosity-capillarity travelling wave criterion. 


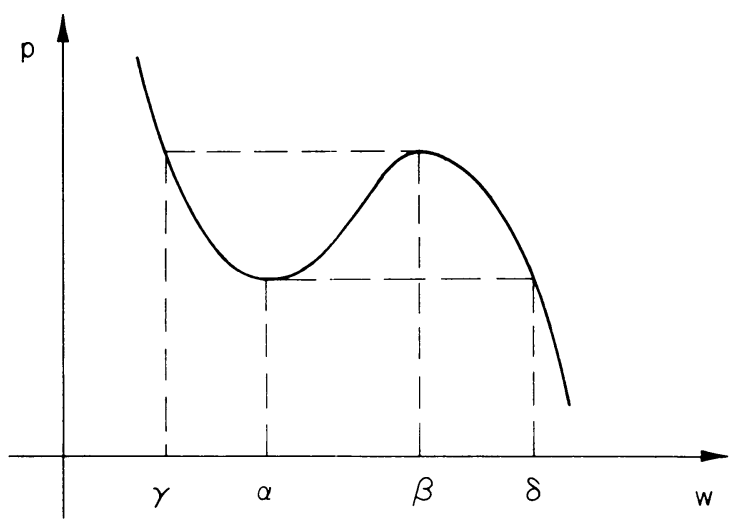

FIGURE 1

In other words, we have to develop some admissibility criterion to single out the "physically correct" solution of (1.1), or better yet, to establish the wellposedness for the Cauchy problem for $(1.1 \mathrm{a}, \mathrm{b})$.

In the context of hyperbolic systems, many admissibility criteria have been proposed. An early example is the Lax shock admissibility criterion [13]. A comprehensive shock admissibility criterion was proposed by Liu [14] which yields a satisfactory solution of the Riemann problem for strictly hyperbolic systems when the waves are of moderate strength. Based on the premise that admissibility should be invariant under translations and dilatations, Dafermos presented, in his recent paper [5], the wave fan admissibility criterion. Dafermos also proposed the entropy rate criterion in [1], and proved in [5] that, for wave fans of moderate strength, the entropy rate criterion and the Liu admissibility condition are equivalent.

A successful criterion for mixed type systems should not only comply with the established criteria for hyperbolic systems for the part of solutions of (1.1) inside a connected component of the hyperbolic region, but should also satisfy physical principles governing phase transitions, for example the Maxwell equal area rule, as well as agree with experimental results. Slemrod [18] suggested, in the context of $(1.1 \mathrm{a}, \mathrm{b})$, the viscosity-capillarity travelling wave criterion, or travelling wave criterion for short, which meets the above standards quite nicely $[8,19,22]$. Based on Korteweg's theory, Slemrod's criterion states that a shock of $(1.1),\left(u_{1}, w_{1}\right),\left(u_{2}, w_{2}\right)$, satisfying, of course, the Rankine-Hugoniot condition, is admissible if

$$
\begin{gathered}
A \frac{d^{2} \hat{w}}{d \zeta^{2}}=-s \frac{d \hat{w}(\zeta)}{d \zeta}-D\left(\frac{d \hat{w}(\zeta)}{d \zeta}\right)^{2}-p(\hat{w})+p\left(w_{1}\right)-s^{2}\left(\hat{w}(\zeta)-w_{1}\right) \\
\hat{w}(-\infty)=w_{1}, \quad \hat{w}(+\infty)=w_{2}, \quad \hat{w}^{\prime}( \pm \infty)=0
\end{gathered}
$$

has a solution, where $A, D$ are constants and $s$ is the speed of the shock. A solution of (1.1) is admissible according to the travelling wave criterion if each discontinuity of the solution is a jump discontinuity and is admissible by the travelling wave criterion. For a recent survey of the travelling wave theory of the dynamics of phase transitions, the reader is referred to [22].

The common approach for solving the Riemann problem (1.1) is to construct the admissible shock and wave curves. If it succeeds, this approach gives us 
centered wave solutions. Discussions using this procedure have been given by R. James [11] and later by M. Shearer [15-17]. L. Hsiao [9, 10] also studied this problem using other admissibility criteria. At this stage, it is natural to ask questions about the existence, uniqueness, and stability of these solutions.

The existence of solutions of (1.1) which are admissible according to the travelling wave criterion (1.2) when $A=1 / 4$ and $D=0$ was established recently by Fan in [7]. Following Slemrod [20], Fan [7] constructed solutions of (1.1) as the $\varepsilon \rightarrow 0+$ limit of solutions of the system

$$
\begin{gathered}
u_{t}+p(w)_{x}=\varepsilon t u_{x x}, \\
w_{t}-u_{x}=\varepsilon t w_{x x}, \\
(u(x, 0), w(x, 0))= \begin{cases}\left(u_{-}, w_{-}\right) & \text {for } x<0, \\
\left(u_{+}, w_{+}\right) & \text {for } x>0 .\end{cases}
\end{gathered}
$$

The above "similarity viscosity" approach was pursued by Kalasnikov [12], Tupciev [23, 24], Dafermos [2, 3, 5], Dafermos and DiPerna [4], Slemrod [20], Slemrod and Tzavaras [21], and Fan [6,7]. For convenience, we shall, by saying that a solution of (1.1) is admissible according to the similarity viscosity admissibility criterion, mean that this solution is constructed by the above similarity viscosity approach. In [7], the author proved the existence of centered wave solutions $(u(x / t), w(x / t))$ of $(1.1)$ which possess one phase change and satisfying the similarity viscosity admissibility criterion under the assumption that

$$
|p(w)| \rightarrow \infty \text {. }
$$

These solutions are also admissible according to the travelling wave criterion with $A=1 / 4$ and $D=0$ if each straight line in the $(w, p)$ plane intersects the graph of $p(w)$ at at most finite points.

In this paper, we assume the following

Assumption 1. Besides (1.1c), $p(w)$ also satisfies

$$
\begin{array}{ll}
p^{\prime \prime}(w)>0 & \text { for } w \leq \alpha, \\
p^{\prime \prime}(w)<0 & \text { for } w \geq \beta .
\end{array}
$$

We shall see in $\S 2$ that under Assumption 1 solutions to the Riemann problem (1.1), satisfying the travelling wave criterion, possess one and only one phase boundary. In the sequel of this paper, when we say solutions of (1.1) we always refer to centered wave solutions of (1.1) which are admissible according to the travelling wave criterion with $A=1 / 4, D=0$.

Under Assumption 1, we shall prove the uniqueness and stability of the solution of (1.1). In $\S 2$, we study the structure of the solution. We devote $\S 3$ to the study of phase boundaries. After this preparation, we shall prove, in $\S 4$, our main result:

Theorem 1.1. (i) (1.1) has a unique solution within the class of centered wave solutions satisfying the travelling wave criterion.

(ii) Let $(u(\xi), w(\xi))$ be the solution of (1.1). For any $\varepsilon>0$ and $\gamma>0$, there is a $\delta>0$ such that if

$$
\left|u_{-}-\bar{u}_{-}\right|+\left|u_{+}-\bar{u}_{+}\right|+\left|w_{-}-\bar{w}_{-}\right|+\left|w_{+}-\bar{w}_{+}\right|<\delta
$$


then

$$
\operatorname{meas}\{\xi \in \mathbb{R}|| u(\xi)-\bar{u}(\xi)|+| w(\xi)-\bar{w}(\xi) \mid \geq \varepsilon\}<\gamma,
$$

where $(\bar{u}(\xi), \bar{w}(\xi))$ is the solution of $(1.1 \mathrm{a}, \mathrm{b})$ with Riemann initial values $\left(\bar{u}_{-}, \bar{w}_{-}\right)$and $\left(\bar{u}_{+}, \bar{w}_{+}\right)$, and 'meas' denotes the Lebesgue measure.

\section{THE STRUCTURE OF SOLUTIONS}

Definition 2.1. A solution of (1.1) is said to be admissible according to the viscosity-capillarity travelling wave criterion (or travelling wave criterion for short) if

(i) at each point $x i_{0}$ of discontinuity of $(u(\xi), w(\xi)),\left(u\left(\xi_{0^{-}}\right), w\left(\xi_{0}-\right)\right)$ and $\left(u\left(\xi_{0}+\right), w\left(\xi_{0}+\right)\right)$ exist, and

(ii) the following boundary value problem has a solution:

$$
\begin{gathered}
\frac{d^{2} \hat{w}(\zeta)}{d \zeta^{2}}=-2 \xi_{0} \frac{d \hat{w}(\zeta)}{d \zeta}+p\left(\xi_{0}-\right)-p(\hat{w}(\zeta))-\xi_{0}^{2}\left(\hat{w}(\zeta)-w\left(\xi_{0}-\right)\right) \\
\hat{w}(-\infty)=w\left(\xi_{0}-\right), \quad \hat{w}(+\infty)=w\left(\xi_{0}+\right), \quad \hat{w}^{\prime}( \pm \infty)=0
\end{gathered}
$$

We first summarize our earlier results on the existence of solutions of (1.1) in the following theorem which is a combination of Corollary 4.7 of [8] and Theorem 4.3 of [7].

Theorem 2.1. (i) Solutions of $(1.1),(u(\xi), w(\xi))$, which are admissible by the viscosity-capillarity travelling wave criterion exist which satisfy the condition that there is a $\xi_{0} \in \mathbb{R}$ such that $w(\xi) \leq \alpha$ for $\xi<\xi_{0}$ and $w(\xi) \geq \beta$ for $\xi>\xi_{0}$.

Lemma 2.2. The boundary value problem (2.1) is equivalent to the following system:

$$
\begin{gathered}
\frac{d \hat{u}(\zeta)}{d \zeta}=-s\left(\hat{u}(\zeta)-u_{1}\right)-p(\hat{w}(\zeta))+p\left(w_{1}\right), \\
\frac{d \hat{w}(\zeta)}{d \zeta}=-s\left(\hat{w}(\zeta)-w_{1}\right)-\hat{u}(\zeta)+u_{1}, \\
(\hat{u}(-\infty), \hat{w}(-\infty))=\left(u_{1}, w_{1}\right), \quad(\hat{u}(\infty), \hat{w}(\infty))=\left(u_{2}, w_{2}\right),
\end{gathered}
$$

where $\left(u_{1}, w_{1}\right),\left(u_{2}, w_{2}\right)$ satisfy the Rankine-Hugoniot condition at $s$.

Proof. Obvious.

In this paper, we always use the following notation:

$$
\lambda(w):=\sqrt{-p^{\prime}(w)} .
$$

Lemma 2.3. Let $(u(\xi), w(\xi))$ be a solution of (1.1) admissible by the travelling wave criterion. Then the following assertions hold:

(i) $w(\xi) \notin(\alpha, \beta)$ for any $\xi \in \mathbb{R}$.

(ii) If $\xi_{0}$ is a point of discontinuity of $(u(\xi), w(\xi))$ and $w\left(\xi_{0} \pm\right) \leq \alpha$ (or $\geq \beta)$, then either

$$
\lambda\left(w\left(\xi_{0}-\right)\right) \geq \xi_{0} \geq \lambda\left(w\left(\xi_{0}+\right)\right)
$$


or

$$
-\lambda\left(w\left(\xi_{0}-\right)\right) \geq \xi_{0} \geq-\lambda\left(w\left(\xi_{0}+\right)\right) .
$$

(iii) If there is a sequence $\left\{\xi_{n}\right\}$ such that $\xi_{n} \rightarrow \xi_{0}-\left(\right.$ or $\left.\xi_{n} \rightarrow \xi_{0}+\right)$, as $n \rightarrow \infty$, and $w\left(\xi_{n}+\right) \neq w\left(\xi_{0}-\right)$ (or $\left.w\left(\xi_{n}-\right) \neq w\left(\xi_{0}+\right)\right)$, then

$$
\xi_{0}= \pm \lambda\left(w\left(\xi_{0}-\right)\right) \quad\left(\text { or } \xi_{0}= \pm \lambda\left(w\left(\xi_{0}+\right)\right)\right) .
$$

(iv) If $w\left(\xi_{0}-\right) \leq \alpha$ and $w\left(\xi_{0}+\right) \geq \beta$, then

$$
\lambda\left(w\left(\xi_{0}-\right)\right) \geq \xi_{0} \geq-\lambda\left(w\left(\xi_{0}+\right)\right)
$$

Proof. (i) The proof is lengthy; therefore we put it in the Appendix.

(ii)-(iv) See the proofs of Proposition 3.6 of [3] and Theorems 5.1, 5.2 of [7], and apply Lemma 2.2 .

We define

$$
f\left(w, w_{1}, s\right):=-p(w)+p\left(w_{1}\right)-s^{2}\left(w-w_{1}\right) .
$$

Lemma 2.4. Suppose (2.1) has a solution.

(i) If $\xi_{0} \geq 0$, then

$$
\int_{w_{1}}^{w} f\left(w, w_{1}, \xi_{0}\right) d w \geq 0 \text { for } w \in\left[w_{1}, w_{2}\right] .
$$

(ii) If $\xi_{0}<0$, then

$$
\int_{w}^{w_{2}} f\left(w, w_{1}, \xi_{0}\right) d w<0 \quad \text { for } w \in\left[w_{1}, w_{2}\right]
$$

Proof. We only prove (i) since the proof of (ii) is similar. Multiplying (2.1) by $\frac{d \hat{w}(\zeta)}{d \zeta}$ and integrating it on $(-\infty, \xi)$, we obtain

$$
\int_{w_{1}}^{w} f\left(w, w_{1}, \xi_{0}\right) d w=\frac{1}{2}\left(\left.\frac{d \hat{w}(\zeta)}{d \zeta}\right|_{\zeta=\xi}\right)^{2}+2 \xi_{0} \int_{-\infty}^{\xi}\left(\frac{d \hat{w}(\zeta)}{d \zeta}\right)^{2} d \zeta \geq 0
$$

In the remainder of this paper, we assume Assumption 1.

Theorem 2.5. Let $\xi_{0}$ be a point of discontinuity of $(u(\xi), w(\xi))$.

(i) If $w\left(\xi_{0}-\right) \leq \alpha$ and $w\left(\xi_{0}+\right) \geq \beta$, then

$$
\lambda\left(w\left(\xi_{0}-\right)\right)>\xi_{0}>-\lambda\left(w\left(\xi_{0}+\right)\right) .
$$

(ia) If further $w\left(\xi_{0}+\right) \neq w_{+}$, then $\xi_{0} \leq \lambda\left(w\left(\xi_{0}+\right)\right)$.

(ib) If $w\left(\xi_{0}-\right) \neq w_{-}$, then $\xi_{0} \geq-\lambda\left(w\left(\xi_{0}-\right)\right)$.

(ii) If $w\left(\xi_{0} \pm\right) \leq \alpha$, then $\xi_{0}<0, w\left(\xi_{0}+\right)<w\left(\xi_{0}-\right)$, and

$$
-\lambda\left(w\left(\xi_{0}-\right)\right)>\xi_{0}>-\lambda\left(w\left(\xi_{0}+\right)\right) .
$$

(iii) If $w\left(\xi_{0} \pm\right) \geq \beta$, then $\xi_{0}>0, w\left(\xi_{0}-\right)>w\left(\xi_{0}+\right)$, and

$$
\lambda\left(w\left(\xi_{0}-\right)\right)>\xi_{0}>\lambda\left(w\left(\xi_{0}+\right)\right) .
$$

Proof. (i) If

$$
\xi_{0} \geq \lambda\left(w\left(\xi_{0}-\right)\right)
$$


then the chord connecting $w\left(\xi_{0}-\right)$ and $w\left(\xi_{0}+\right)$ lies below the graph of $p(w)$ which violates Lemma 2.4(i). Thus

$$
\xi_{0}<\lambda\left(w\left(\xi_{0}-\right)\right)
$$

The other half of $(2.11)$ can be proved similarly.

Let $w\left(\xi_{0}+\right) \neq w_{+}$and suppose, for contradiction, that

$$
\xi_{0}>\lambda\left(w\left(\xi_{0}+\right)\right) \text {. }
$$

Then, by Lemma $2.3,(u(\xi), w(\xi))$ is constant in $\left(\xi_{0}, \xi_{1}\right)$ for some $\xi_{1}>\xi_{0}$. We denote the supremum of such $\xi_{1}$ by $\xi_{2}$, and hence

$$
\xi_{2}>\xi_{0}
$$

and

$$
w\left(\xi_{2}-\right)=w\left(\xi_{0}+\right)
$$

If $\xi_{2}$ is a point of discontinuity of $(u(\xi), w(\xi))$, then $\xi_{2} \leq \lambda\left(w\left(\xi_{2}-\right)\right)$. If $\xi_{2}$ is a point of continuity, then, by the definition of $\xi_{2}$ and Lemma $2.3, \xi_{2}=$ $\lambda\left(w\left(\xi_{2}\right)\right)$. In both cases, we have

$$
\xi_{2} \leq \lambda\left(w\left(\xi_{2}-\right)\right)=\lambda\left(w\left(\xi_{0}+\right)\right)<\xi_{0},
$$

which contradicts $(2.15)$.

The proof for (ib) is similar to that of (ia).

(ii) By Lemma 2.3, we have either

$$
\lambda\left(w\left(\xi_{0}+\right)\right) \leq \xi_{0} \leq \lambda\left(w\left(\xi_{0}-\right)\right)
$$

or

$$
-\lambda\left(w\left(\xi_{0}+\right)\right) \leq \xi_{0} \leq-\lambda\left(w\left(\xi_{0}-\right)\right) .
$$

We claim that $(2.17 \mathrm{a})$ does not hold. Indeed, if otherwise, $\xi_{0}>0$. Since $w\left(\xi_{0} \pm\right) \leq \alpha$, there is an $\eta>\xi_{0}>0$ such that $w(\eta-) \leq \alpha<\beta \leq w(\eta+)$. It follows from (i) that

$$
\eta<\lambda(w(\eta-))
$$

By Lemma 2.3(iii), $w(\xi)$ will be constant in $\left(\eta_{1}, \eta\right)$. We let $\eta_{2}$ be the infimum of such $\eta_{1}$ and therefore

$$
\eta>\eta_{2} \geq \xi_{0}>0
$$

If $\eta_{2}$ is a point of discontinuity of $(u(\xi), w(\xi))$, then $w\left(\eta_{2} \pm\right) \leq \alpha$ and hence $\eta_{2} \geq \lambda\left(\eta_{2}+\right)$. If $\eta_{2}$ is a point of continuity of $(u(\xi), w(\xi))$, then, by the definition of $\eta_{2}, \eta_{2}=\lambda\left(w\left(\eta_{2}\right)\right)$. In both cases, we have

$$
\eta_{2} \geq \lambda\left(w\left(\eta_{2}+\right)\right)=\lambda(w(\eta-))>\eta
$$

which contradicts $(2.18)$. Thus only $(2.17 \mathrm{~b})$ holds. Equality in $(2.17 \mathrm{~b})$ cannot hold since $p(w)$ is convex and

$$
\xi_{0}^{2}=-\frac{p\left(w\left(\xi_{0}+\right)\right)-p\left(w\left(\xi_{0}-\right)\right)}{w\left(\xi_{0}+\right)-w\left(\xi_{0}-\right)} .
$$

Therefore $(2.10)$ is proved. $w\left(\xi_{0}+\right)<w\left(\xi_{0}-\right)$ follows easily from the convexity of $p(w)$ and (2.10).

(iii) The proof for (iii) is similar to that of (ii). 
Theorem 2.6. (i) If there is a sequence $\left\{\xi_{n}\right\}$ such that $\xi_{n} \rightarrow \xi_{0}-$, as $n \rightarrow \infty$, and $w\left(\xi_{n}+\right) \neq w\left(\xi_{0}-\right)$, and

(ia) if $w\left(\xi_{0}-\right) \leq \alpha$, then

$$
\xi_{0}=-\lambda\left(w\left(\xi_{0}-\right)\right)
$$

(ib) if $w\left(\xi_{0}-\right) \geq \beta$, then

$$
\xi_{0}=\lambda\left(w\left(\xi_{0}-\right)\right) .
$$

(ii) If there is a sequence $\left\{\xi_{n}\right\}$ such that $\xi_{n} \rightarrow \xi_{0}+$, as $n \rightarrow \infty$, and $w\left(\xi_{n}-\right) \neq$ $w\left(\xi_{0}+\right)$, and

(iia) if $w\left(\xi_{0}+\right) \leq \alpha$, then

$$
\xi_{0}=-\lambda\left(w\left(\xi_{0}+\right)\right)
$$

(iib) if $w\left(\xi_{0}+\right) \geq \beta$, then

$$
\xi_{0}=\lambda\left(w\left(\xi_{0}+\right)\right) .
$$

Proof. We prove (ia) only since the proofs for the rest of the theorem are similar.

In this case, $\xi_{0}= \pm \lambda\left(w\left(\xi_{0}-\right)\right)$ as asserted by Lemma 2.3. Assume, for contradiction, $w\left(\xi_{0}-\right) \leq \alpha$ and $\xi_{0}>0$. Then there is an $\eta \in \mathbb{R}$ such that $\eta \geq \xi_{0}$ and $w(\eta-) \leq \alpha<\beta \leq w(\eta+)$. By Theorem 2.5(i), $\eta=\xi_{0}$ cannot hold and hence $\eta>\xi_{0}$. By Lemma 2.3, $w(\xi)$ will be constant in $\left(\eta_{1}, \eta\right)$ for some $\eta_{1}<\eta$. Similar to what we did in the proof of Theorem 2.5(ii), we let $\eta_{2}$ be the infimum of such $\eta_{1}$ and therefore

$$
\eta>\eta_{2} \geq \xi_{0}>0
$$

Theorem 2.5(ii) says that $\eta_{2}$ cannot be a point of discontinuity of $(u(\xi), w(\xi))$ or otherwise $\eta_{2}<0$ which is prohibited by (2.21). Thus $\eta_{2}$ is a point of continuity of $(u(\xi), w(\xi))$. By the definition of $\eta_{2}$, we can see that $\eta_{2}=$ $\lambda\left(w\left(\eta_{2}\right)\right)$. Therefore,

$$
\eta_{2}=\lambda\left(w\left(\eta_{2}+\right)\right)=\lambda(w(\eta-))>\eta,
$$

which contradicts $(2.21)$.

Theorem 2.7. Let $(u(\xi), w(\xi))$ be a solution of $(1.1)$.

(i) There is one and only one phase boundary in the solution, i.e., there is a $\xi_{0} \in \mathbb{R}$ such that $w(\xi)>\beta$ for $\xi>\xi_{0}$, and $w(\xi)<\alpha$ for $\xi<\xi_{0}$

(ii) In the region $w \leq \alpha$, solutions of $(1.1),(u(\xi), w(\xi))$, consist of either a constant state $\left(u_{-}, w_{-}\right)$or two constant states $\left(u_{-}, w_{-}\right)$and $\left(u_{1}, w_{1}\right)$ joined by a shock with speed $s_{1}<0$ or a backward rarefaction wave with $\left(u_{-}, w_{-}\right)$on its left.

(iii) In the region $w \geq \beta$, solutions of $(1.1),(u(\xi), w(\xi))$, consist of either a constant state $\left(u_{+}, w_{+}\right)$or two constant states $\left(u_{+}, w_{+}\right)$and $\left(u_{2}, w_{2}\right)$ joined by a shock with speed $s_{3}>0$ or a forward rarefaction wave with $\left(u_{+}, w_{+}\right)$on its right.

(iv) $\left(u_{1}, w_{1}\right)$ and $\left(u_{2}, w_{2}\right)$ are joined by a shock, i.e., the phase boundary. Proof. We prove (i) and (ii) only since that of (iii) is similar, and (iv) follows immediately.

(i) Suppose that there are more than one phase boundaries in a solution $(u(\xi), w(\xi))$. Then there are at least three phase boundaries because $w_{-}<$ $\alpha<\beta<w_{+}$. More precisely, there are points of discontinuity $\xi_{0}, \xi_{1}, \xi_{2} \in \mathbb{R}$ 
of $(u(\xi), w(\xi))$ such that $w\left(\xi_{j}-\right)<\alpha<\beta<w\left(\xi_{j}+\right), j=0,2$, and $w\left(\xi_{1}+\right)<$ $\alpha<\beta<w\left(\xi_{1}-\right)$. Without loss of generality, we assume $\xi_{0}<\xi_{1}<\xi_{2}$ and that there are no other points of dicontinuity of $(u(\xi), w(\xi))$ between $\xi_{0}$ and $\xi_{2}$. At least two of $\xi_{j}, j=0,1,2$, are nonnegative or nonpositive. We consider the case $0 \leq \xi_{1}<\xi_{2}$ only, since the proof for the other cases are similar. For the point $\xi_{1}$, we know from (2.8) in the proof of Lemma 2.4 that

$$
\int_{w\left(\xi_{1}+\right)}^{w\left(\xi_{1}-\right)} f\left(w, w\left(\xi_{1}+\right), \xi_{1}\right) d w \leq 0 .
$$

Theorems 2.5(ii) and 2.6(ii) imply that $(u(\xi), w(\xi))$ is constant for $\xi \in\left(\xi_{1}, \xi_{2}\right)$ since $\xi_{1} \geq 0$. Thus $w\left(\xi_{2}-\right)=w\left(\xi_{1}+\right)$. Lemma 2.4 then leads to

$$
\int_{w\left(\xi_{1}+\right)}^{w\left(\xi_{2}+\right)} f\left(w, w\left(\xi_{1}+\right), \xi_{2}\right) d w>0 .
$$

From (2.22) and (2.23), we know, by an inspection on the graph of $p(w)$, that $\xi_{1}>\xi_{2}>0$, which is a contradiction.

(ii) Suppose $(u(\xi), w(\xi))$ has a point $\xi_{0}$ of discontinuity with $w\left(\xi_{0} \pm\right) \leq \alpha$. By Theorem $2.5, \xi_{0}<0$. We define a subset of $\mathbb{R}$ by

$$
A:=\left\{\xi>\xi_{0} \mid w(\xi) \neq w\left(\xi_{0}+\right), w(\xi \pm) \leq \alpha\right\} .
$$

We claim that $A$ is empty. Indeed, if otherwise, we can define

$$
\eta:=\inf A \geq \xi_{0} .
$$

If $\eta=\xi_{0}$, then there is a sequence $\xi_{n} \rightarrow \eta+=\xi_{0}+$ such that $w\left(\xi_{n}+\right) \neq$ $w\left(\xi_{0}+\right)$. By Theorem 2.6,

$$
\xi_{0}=-\lambda\left(w\left(\xi_{0}+\right)\right),
$$

which is impossible in view of Theorem 2.5. If $\eta>\xi_{0}$, then $w(\eta-)=w\left(\xi_{0}+\right)$. If, further, $w(\eta-) \neq w(\eta+)$, then, by Theorem 2.5 ,

$$
\eta<-\lambda(w(\eta-))=-\lambda\left(w\left(\xi_{0}+\right)\right)<\xi_{0},
$$

which contradicts the definition of $\eta$ in (2.25). If $w(\eta-)=w(\eta+)$, then there is a sequence $\xi_{n} \rightarrow \eta+$ as $n \rightarrow \infty$ such that $w\left(\xi_{n}+\right) \neq w(\eta+)$. Again by Theorem 2.6, we obtain

$$
\eta=-\lambda(w(\eta \pm))=-\lambda\left(w\left(\xi_{0}+\right)\right)<\xi_{0},
$$

which is also impossible. Thus, $A$ is empty, which simply says that $(u(\xi), w(\xi))$ must be constant for $\xi \in\left(\xi_{0}, s_{2}\right)$ for some $s_{2}$ such that $w\left(s_{2}+\right) \geq \beta$.

Similarly, we can prove that $(u(\xi), w(\xi))$ is constant for $\xi \in\left(-\infty, \xi_{0}\right)$.

From the above results, we can see that a solution $(u(\xi), w(\xi))$ of $(1.1)$ consists of a shock $\xi=s_{2}$, such that $w_{1}:=w\left(s_{2}-\right) \leq \alpha<\beta \leq w\left(s_{2}+\right)=$ : $w_{2}$, and two constant states $\left(u_{-}, w_{-}\right)$and $\left(u_{+}, w_{+}\right) .\left(u_{-}, w_{-}\right)$is joined to $\left(u\left(s_{2}-\right), w\left(s_{2}-\right)\right)$ by either a backward shock $\xi=s_{1}<0$ if $w\left(s_{2}-\right)<w_{-}$ or a backward rarefaction wave if $w\left(s_{2}-\right)>w_{-} .\left(u_{+}, w_{+}\right)$is connected to $\left(u\left(s_{2}+\right), w\left(s_{2}+\right)\right)$ by either a forward shock $\xi=s_{3}>0$ if $w\left(s_{2}+\right)>w_{+}$or a 
forward rarefaction wave if $w\left(s_{2}+\right)<w_{+}$. Thus, we can denote a solution of (1.1), for simplicity, by $\left\{w_{1}, w_{2}, s_{2}\right\}$.

\section{THE PHASE BOUNDARY}

Let $\xi=s$ be a point of discontinuity of $(u(\xi), w(\xi))$. For notational simplicity, we denote $\left(u_{1}, w_{1}\right):=(u(s-), w(s-)),\left(u_{2}, w_{2}\right):=(u(s+), w(s+))$. Then we have the Rankine-Hugoniot conditions:

$$
\begin{gathered}
-s\left(u_{2}-u_{1}\right)+p\left(w_{2}\right)-p\left(w_{1}\right)=0, \\
-s\left(w_{2}-w_{1}\right)-\left(u_{2}-u_{1}\right)=0 .
\end{gathered}
$$

The speed $s$ of the shock is determined by

$$
s^{2}=-\frac{p\left(w_{2}\right)-p\left(w_{1}\right)}{w_{2}-w_{1}} .
$$

We call $w_{1} \rightarrow w_{2}$ a connection if (2.1) has a solution. In this section, we devote ourselves to connections $w_{1} \rightarrow w_{2}$, where $w_{1} \leq \alpha<\beta \leq w_{2}$, which are called phase boundaries.

Lemma 3.1. Let $\alpha>w_{1} \rightarrow w_{2}>\beta$ be a connection with speed $s$.

(i) If $s=0$, then $w_{1}=m$ and $w_{2}=M$, where $m<\alpha$ and $M>\beta$ are Maxwell constants defined by

$$
p(m)=p(M), \quad \int_{m}^{M}(p(w)-p(m)) d w=0 .
$$

(ii) If $s \geq 0$, then $w_{1} \leq m$.

(iii) If $s<0$, then $w_{2}>M$.

Proof. (i) is proved in [15]. (ii) and (iii) follow from Lemma 2.4.

Lemma 3.2. Let $s \geq 0$ in (2.1). Then the connected component of the unstable manifold of $\left(w_{1}, 0\right)$ in the upper half phase plane containing $\left(w_{1}, 0\right)$ is unique and is denoted by $\Gamma_{1}\left(w_{1}, w_{2}\right)$.

The connected component of the stable manifold of $\left(w_{2}, 0\right)$ in the upper half phase plane containing $\left(w_{2}, 0\right)$ is unique and is denoted by $\Gamma_{2}\left(w_{1}, w_{2}\right)$.

Proof. We can rewrite (2.1) as

$$
\begin{gathered}
v \frac{d v}{d \hat{w}}=-2 s v+f\left(\hat{w}, w_{1}, s\right), \\
\frac{d \hat{w}}{d \zeta}=v,
\end{gathered}
$$

where $f\left(w, w_{1}, s\right):=-p(w)+p\left(w_{1}\right)-s^{2}\left(w-w_{1}\right)$. Along the trajectories of (3.5) which are in the upper half phase plane, $\hat{w}^{\prime}(\zeta)=v \geq 0$. Thus, we can parametrize this part of the trajectories by $v(w)$.

Suppose (3.5) has two unstable manifolds leaving $\left(w_{1}, 0\right)$ into the upper half phase plane. We denote these two manifolds by $v(w)$ and $\bar{v}(w)$ respectively. If $v\left(w_{0}\right)=\bar{v}\left(w_{0}\right)>0$ for some $w_{0}>w_{1}$, then the uniqueness of (3.5) in half plane $v>0$ implies $v(w)=\bar{v}(w)$. Thus, without loss of generality, we can assume

$$
v(w)>\bar{v}(w)
$$


for $w \in\left(w_{1}, w_{1}+\mu\right)$ for some $\mu>0$. Then it follows from (3.5) that

$$
v \frac{d v}{d w}-\bar{v} \frac{d \bar{v}}{d w}=-2 s(v-\bar{v}) \text {. }
$$

Integrating (3.7) from $w_{1}$ to $w$, we obtain, by virtue of (3.6) and $s \geq 0$, that

$$
0<v^{2}(w)-\bar{v}^{2}(w)=-2 s \int_{w_{1}}^{w}(v(w)-\bar{v}(w)) d w \leq 0
$$

for $w \in\left(w_{1}, w_{1}+\mu\right)$, which is the desired contradiction.

The proof for the uniqueness of the stable manifold entering $\left(w_{2}, 0\right)$ is similar.

Lemma 3.3. Let $w_{1} \leq \alpha, w_{2} \geq \beta$ satisfy

$$
s \leq \lambda\left(w_{2}\right)
$$

where $s^{2}$ is determined by (3.2). Then $w_{1} \rightarrow w_{2}$ is a connection with $s \geq 0$ if and only if $\Gamma_{1}\left(w_{1}, w_{2}\right)=\Gamma_{2}\left(w_{1}, w_{2}\right)$.

Proof. It is clear that if $\Gamma_{1}\left(w_{1}, w_{2}\right)=\Gamma_{2}\left(w_{1}, w_{2}\right)$, then $w_{1} \rightarrow w_{2}$ is a connection.

Conversely, if $w_{1} \rightarrow w_{2}$ is a connection, then the connecting trajectory $\hat{w}(\zeta)$ has to cross the $w$-axis at some $\left(w_{0}, 0\right)$ for some $w_{0} \geq w_{2}$. On the other hand (3.8) and Assumption 1 together with (2.1) imply that $w_{0} \leq w_{2}$. Thus $\Gamma_{1}\left(w_{1}, w_{2}\right)=\Gamma_{2}\left(w_{1}, w_{2}\right)$.

We denote the manifolds $\Gamma_{1}\left(w_{1}, w_{2}\right)$ and $\Gamma_{2}\left(w_{1}, w_{2}\right)$ by $v_{1}(w, s)$ and $v_{2}(w, s)$ respectively to specify the dependence of $v_{1}, v_{2}$ on $s$.

The proof of the following lemma was first given by M. Shearer [16]:

Lemma 3.4. (i)

(ii)

$$
\frac{\partial v_{1}}{\partial s}(w, s)<0
$$

$$
\frac{\partial v_{2}}{\partial s}(w, s)>0
$$

Proof. (i) It follows from (3.5) that

$$
\frac{d v}{d w}=-2 s+\frac{f\left(w, w_{1}, s\right)}{v} .
$$

As $w \rightarrow w_{1}$ or $w_{2}, f\left(w, w_{1}, s\right) \rightarrow 0$ and $v \rightarrow 0$. A simple computation based on (3.9) shows that

$$
\left.\frac{\partial v}{\partial w}\right|_{w=w_{1}}=-s+\lambda\left(w_{1}\right)
$$

and hence

$$
\left.\frac{\partial^{2} v_{1}}{\partial s \partial w}\right|_{w=w_{1}}=-1 .
$$

Since $v_{1}\left(w_{1}\right) \equiv 0$ and thus $\left.\frac{\partial v_{1}}{\partial s}\right|_{w=w_{1}}=0$,

$$
\frac{\partial v_{1}}{\partial s}(w, s)<0
$$

for $w>w_{1}$ and near $w_{1}$. 
On the other hand, by integrating (3.5) from $w_{1}$ to some $w$, we obtain

$$
\frac{v_{1}^{2}(w)}{2}=\int_{w_{1}}^{w}\left[-2 s v_{1}(\theta)+f\left(\theta, w_{1}, s\right)\right] d \theta,
$$

$$
v_{1}(w) \frac{\partial v_{1}}{\partial s}(w, s)=2 \int_{w_{1}}^{w}\left[-v_{1}(\theta)-s \frac{\partial v_{1}}{\partial s}(\theta, s)-s\left(\theta-w_{1}\right)\right] d \theta \equiv 2 g(w) .
$$

Now, we claim $\frac{\partial v_{1}}{\partial s}(w, s)<0$. Indeed, if otherwise, there is $w^{*}>w_{1}$ such that $\frac{\partial v_{1}}{\partial s}\left(w^{*}, s\right)=0$ and $\frac{\partial v_{1}}{\partial s}(w, s)<0$ for all $w \in\left(w_{1}, w^{*}\right)$. In other words, $g\left(w_{1}\right)=g\left(w^{*}\right)=0$ and $g(w)<0$ for all $w \in\left(w_{1}, w^{*}\right)$. Thus

$$
g^{\prime}\left(w^{*}\right) \geq 0
$$

However,

$$
g^{\prime}\left(w^{*}\right)=-v_{1}\left(w^{*}\right)-s\left(w^{*}-w_{1}\right)<0,
$$

which contradicts (3.15). Thus $\frac{\partial v_{1}}{\partial s}(w, s)<0$.

(ii) The proof is similar to that of (i).

We define

$$
\begin{gathered}
w_{2}\left(w_{1}, s\right):=\max \left\{w \geq \beta \mid p(w)=p\left(w_{1}\right)-s^{2}\left(w-w_{1}\right)\right\} \\
w_{3}\left(w_{1}, s\right):=\min \left\{w \geq \beta \mid p(w)=p\left(w_{1}\right)-s^{2}\left(w-w_{1}\right)\right\}
\end{gathered}
$$

Theorem 3.5. Let $\alpha>w_{1} \rightarrow w_{2}>\beta$ be a connection with speed $\lambda\left(w_{2}\right)>s \geq 0$. Then any $w^{*} \in\left[w_{3}\left(w_{1}, s\right), w_{2}\right)$ cannot be connected to $w_{1}$.

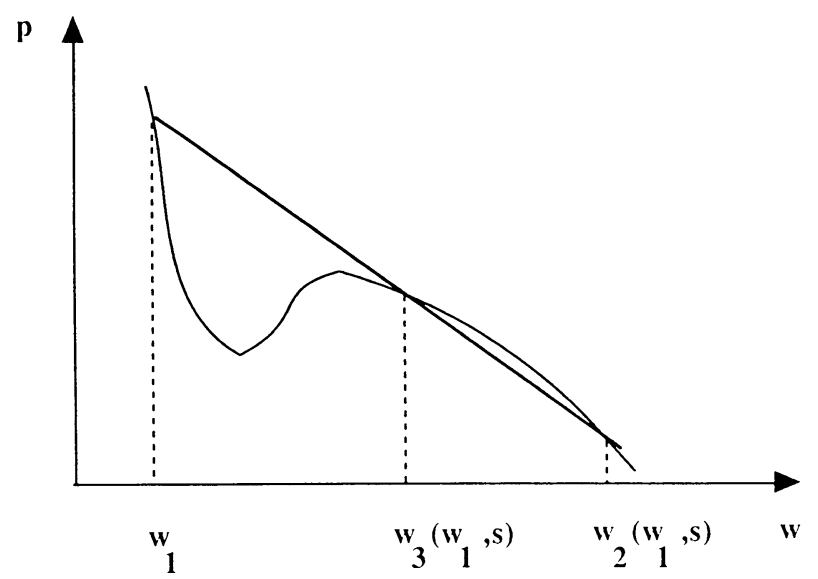

FIGURE 2

Proof. Assume the contrary, i.e. there is a $w^{*} \in\left[w_{3}\left(w_{1}, s\right), w_{2}\right)$ such that $w_{1} \rightarrow w^{*}$ is a connection. An inspection on the graph of $p(w)$ tells us that

$$
s^{* 2}=-\frac{p\left(w^{*}\right)-p\left(w_{1}\right)}{w^{*}-w_{1}}<s^{2} .
$$


By Lemma 2.4, $0 \leq s^{*}<s$. Lemma 3.3 implies that

$$
v_{1}\left(w, s^{*}\right)>v_{1}(w, s)>0
$$

for $w \in\left[w_{3}\left(w_{1}, s\right), w_{2}\right)$. It is clear from (3.18) that $f\left(w, w_{1}, s^{*}\right)>0$ for $w \geq w_{2}$ and hence $v_{1}\left(w, s_{3}\right)>0$ for $w \geq w_{0}(s)$. That is, $w_{1} \rightarrow w^{*}$ is not a connection.

Theorem 3.6. Let $\alpha>w_{1} \rightarrow w_{2}>\beta$ be a connection with speed $0 \leq s \leq \lambda\left(w_{2}\right)$. If $\alpha>\bar{w}_{1} \rightarrow \bar{w}_{2}>\beta$ is a connection with speed $\bar{s} \geq 0$ and if $\bar{w}_{1}<w_{1}$, then $\bar{s}>s$.

Proof. Consider the unstable manifold $\Gamma_{1}\left(\bar{w}_{1}, \bar{w}_{2}\right)$ leaving $\left(\bar{w}_{1}, 0\right)$ into the upper half phase plane which we shall denote by $\bar{v}(w)$. We have

$$
\bar{v} \frac{d \bar{v}}{d w}=-2 \bar{s} \bar{w}-p(w)+p\left(\bar{w}_{1}\right)-\bar{s}^{2}\left(w-\bar{w}_{1}\right) .
$$

Since $\bar{w}_{1} \rightarrow \bar{w}_{2}$ is a connection, $\Gamma_{1}\left(\bar{w}_{1}, \bar{w}_{2}\right)$ must cross the $w$-axis at $\left(w_{0}, 0\right)$ for some $w_{0} \leq w_{2}\left(\bar{w}_{1}, \bar{s}\right)$, where the unstable manifold $\Gamma_{1}\left(w_{1}, w_{2}\left(w_{1}, \bar{s}\right)\right)$, parametrized by $v(w)$ satisfies

$$
v \frac{d v}{d w}=-2 \bar{s} v-p(w)+p\left(w_{1}\right)-\bar{s}^{2}\left(w-w_{1}\right) .
$$

Subtracting (3.20) from (3.21), we obtain

$$
v \frac{d v}{d w}-\bar{v} \frac{d \bar{v}}{d w}=-2 \bar{s}(v-\bar{v})+p\left(w_{1}\right)-p\left(\bar{w}_{1}\right)-\bar{s}^{2}\left(\bar{w}_{1}-w_{1}\right) .
$$

If $\bar{s} \geq \lambda\left(w_{1}\right)$, then there is nothing to be proved since $s<\lambda\left(w_{1}\right)$ by Theorem 2.5. Thus, without loss of generality, we assume $\bar{s}^{2}<-p^{\prime}\left(w_{1}\right)$. Then

$$
\bar{s}^{2}<\min \left(-p^{\prime}\left(\bar{w}_{1}\right),-p^{\prime}\left(w_{1}\right)\right)<-\frac{p\left(\bar{w}_{1}\right)-p\left(w_{1}\right)}{\bar{w}_{1}-w_{1}} .
$$

The last inequality comes from the convexity of $p(w)$ in the region $w<\alpha$. Applying (3.23) to (3.22), we get

$$
v \frac{d v}{d w}-\bar{v} \frac{d \bar{v}}{d w}<-2 \bar{s}(v-\bar{v}) .
$$

Now, we claim that $v(w), \bar{v}(w)$ do not intersect. Since $\bar{v}\left(w_{1}\right)>v\left(w_{1}\right)=0$, if the contrary of our claim holds, there will be $w^{*}>\bar{w}_{1}$ such that $\bar{v}(w)>$ $v(w)$ for $w \in\left[w_{1}, w^{*}\right)$ and $v\left(w^{*}\right)=\bar{v}\left(w^{*}\right)$. Thus, $v^{\prime}\left(w^{*}\right) \geq \bar{v}^{\prime}\left(w^{*}\right)$, which contradicts the consequence of (3.24)

$$
\left.v\left(w^{*}\right) \frac{d(v-\bar{v})}{d w}\right|_{w=w^{*}}<0
$$

and hence our claim. Therefore, $v(w)$ must meet the $w$-axis at some $\left(w_{0}, 0\right)$, where $w_{0} \in\left(w_{1}, w_{2}\left(\bar{w}_{1}, \bar{s}\right)\right)$. If $s \geq \bar{s}$, then, by Lemma 3.3, $\Gamma_{1}\left(\bar{w}_{1}, \bar{w}_{2}\right)$ will meet the $v$-axis at some $w \in\left(w_{1}, w_{2}\left(\bar{w}_{1}, \bar{s}\right)\right)$ and hence cannot join $w_{2}\left(w_{1}, s\right)>w_{2}\left(\bar{w}_{1}, \bar{s}\right)$. This simply says that $w_{1} \rightarrow w_{2}$ is not a connection with $0 \leq s \leq \lambda\left(w_{2}\right)$. This contradiction completes our proof.

For connections $\alpha>w_{1} \rightarrow w_{2}>\beta$ with $s \leq 0$, we have the following similar results. 
Theorem 3.7. (i) Let $w_{1} \rightarrow w_{2}$ be a connection with $-\lambda\left(w_{1}\right) \leq s \leq 0$. Then any $w^{*} \in\left(w_{1}, w_{0}\left(w_{2}, s\right)\right)$ cannot be connected to $w_{2}$, where

$$
w_{0}\left(w_{2}, s\right):=\max \left\{w<\alpha \mid p(w)=p\left(w_{2}\right)-s^{2}\left(w-w_{2}\right)\right\} .
$$

(ii) Let $\alpha>w_{1} \rightarrow w_{2}>\beta$ be a connection with speed $0 \geq s \geq-\lambda\left(w_{2}\right)$. If $\alpha>\bar{w}_{1} \rightarrow \bar{w}_{2}>\beta$ is a connection with speed $\bar{s} \leq 0$ and if $\bar{w}_{2}>w_{2}$, then $\bar{s}<s$.

Proof. Assume $w_{1} \rightarrow w_{2}$ is a connection with $s \leq 0$. Then (2.1) has a solution. We can replace $w_{1}$ in $(2.1)$ by $w_{2}$, since we have the Rankine-Hugoniot conditions (3.1), to obtain

$$
\begin{gathered}
\frac{d^{2} \hat{w}}{d \zeta^{2}}=-2 s \frac{d \hat{w}(\zeta)}{d \zeta}-p(\hat{w})+p\left(w_{2}\right)-s^{2}\left(\hat{w}-w_{2}\right), \\
\hat{w}(-\infty)=w_{1}, \quad \hat{w}(+\infty)=w_{2}, \quad \hat{w}^{\prime}( \pm \infty)=0 .
\end{gathered}
$$

After applying the following transformations

$$
\zeta \mapsto-\zeta, \quad \hat{w} \mapsto-\hat{w}
$$

in (2.1), (3.26) becomes

$$
\begin{gathered}
\frac{d^{2} \hat{w}}{d \zeta^{2}}=-2(-s) \frac{d \hat{w}(\zeta)}{d \zeta}-P(\hat{w})+P\left(-w_{2}\right)-s^{2}\left(\hat{w}-\left(-w_{2}\right)\right), \\
\hat{w}(-\infty)=-w_{2}, \quad \hat{w}(+\infty)=-w_{1}, \quad \hat{w}^{\prime}( \pm \infty)=0,
\end{gathered}
$$

where $P(w):=-p(-w)$ also satisfies Assumption 1. Now we can apply Theorems $3.5,3.6$ to $(3.28)$ to complete the proof of our theorem.

\section{The UNIQUENeSS AND STABILITY OF THE SOLUTION OF (1.1)}

Lemma 4.1. (1.1) has a solution $\left\{w_{1}, w_{2}, s_{2}\right\}$ if and only if the following conditions hold:

$$
F\left(w_{1}, w_{2}, s_{2}\right)=u_{+}-u_{-},
$$

where $w_{1} \rightarrow w_{2}$ is a connection with speed $s_{2}$ and $w_{1} \leq \alpha<\beta \leq w_{2}$,

$$
\begin{aligned}
F\left(w_{1}, w_{2}, s_{2}\right):= & -s_{1}\left(w_{1}-w_{-}\right) H\left(w_{-}-w_{1}\right) \\
& +H\left(w_{1}-w_{-}+\right) \int_{w_{-}}^{w_{1}} \lambda(w) d w \\
& -s_{2}\left(w_{2}-w_{1}\right)-H\left(w_{2}-w_{+}\right) s_{3}\left(w_{+}-w_{2}\right) \\
& +H\left(w_{+}-w_{2}+\right) \int_{w_{+}}^{w_{2}} \lambda(w) d w,
\end{aligned}
$$

where $H(w)$ is the Heaviside function and

$$
s_{k}^{2}:=-\frac{p\left(w_{k}\right)-p\left(w_{k-1}\right)}{w_{k}-w_{k-1}}, \quad k=1,2,3,
$$

$w_{0}:=w_{-}, w_{3}:=w_{+} ;$

$$
\begin{array}{cc}
s_{1}<0, \quad s_{3}>0, & s_{1}<s_{2}<s_{3}, \\
-\lambda\left(w_{1}\right)<s_{1}<-\lambda\left(w_{-}\right), & \lambda\left(w_{+}\right)<s_{3}<\lambda\left(w_{2}\right),
\end{array}
$$




$$
\begin{gathered}
-\lambda\left(w_{2}\right)<s_{2}<\lambda\left(w_{1}\right), \\
s_{2} \leq \lambda\left(w_{2}\right) \quad \text { if } w_{2} \neq w_{+}, \\
s_{2} \geq-\lambda\left(w_{1}\right) \quad \text { if } w_{1} \neq w_{-} .
\end{gathered}
$$

Proof. Suppose (1.1) has a solution, denoted by $\left\{w_{1}, w_{2}, s_{2}\right\}$. From the discussion following Theorem 2.7, $\left\{w_{1}, w_{2}, s_{2}\right\}$ has the following structure: When $w_{1}:=w\left(s_{2}-\right)<w_{-}$, the solution $(u(\xi), w(\xi))$, for $\xi<s_{2}$, consists of a shock of speed $s_{1}<0$ joining constant states $\left(u_{-}, w_{-}\right)$and $\left(u_{1}, w_{1}\right)$. When $w_{1} \geq w_{-}$, the solution $(u(\xi), w(\xi))$, for $\xi<s_{2}$, consists of a backward rarefaction wave connecting $\left(u_{-}, w_{-}\right)$and $\left(u_{1}, w_{1}\right)$. Therefore,

$$
u_{1}-u_{-}=-H\left(w_{-}-w_{1}\right) s_{1}\left(w_{1}-w_{-}\right)+H\left(w_{1}-w_{-}\right) \int_{w_{-}}^{w_{1}} \lambda(w) d w,
$$

where

$$
s_{1}=-\frac{p\left(w_{1}\right)-p\left(w_{-}\right)}{w_{1}-w_{-}} .
$$

Similarly, we have

$$
u_{2}-u_{1}=-s_{2}\left(w_{2}-w_{1}\right)
$$

and

$$
u_{+}-u_{2}=-H\left(w_{2}-w_{+}\right) s_{3}\left(w_{+}-w_{2}\right)+H\left(w_{+}-w_{2}\right) \int_{w_{+}}^{w_{2}} \lambda(w) d w
$$

where

$$
s_{k}=-\frac{p\left(w_{k}\right)-p\left(w_{k-1}\right)}{w_{k}-w_{k-1}},
$$

$k=2,3$, and $w_{3}:=w_{+} \cdot(4.2)$ follows easily from (4.5), (4.7), and (4.9). The constraints (4.4) follow from Theorems 2.5 and Lemma 2.4. The necessity is proved.

By Theorem 3.3 of [8], the shock solution $\left(u_{-}, w_{-}\right),\left(u_{1}, w_{1}\right)\left(\left(u_{2}, w_{2}\right)\right.$, $\left.\left(u_{+}, w_{+}\right)\right)$is admissible if $w_{1}<w_{-}\left(w_{2}>w_{+}\right)$. Thus, conditions in Lemma 4.1 simply say that $\left\{w_{1}, w_{2}, s_{2}\right\}$ satisfies the initial conditions $(1.1 \mathrm{c}, \mathrm{d})$, is admissible, and hence is a solution of (1.1). Thus the sufficiency is also proved.

In view of Theorems 3.4 and 3.8 , we can also write $F\left(w_{1}, w_{2}, s_{2}\right)$ as $G\left(w_{1}, s_{2}\right)$ for $s_{2} \geq 0$ and $J\left(w_{2}, s_{2}\right)$ for the case $s_{2} \leq 0$.

Remark 4.1. When we consider (4.1) as a necessary condition for (1.1) to have a solution, it will be more convenient to extend the domain of definition of $G\left(w_{1}, s_{2}\right)\left(J\left(w_{2}, s_{2}\right)\right)$ to include $s_{2}=s_{3} \quad\left(s_{2}=s_{1}\right)$. If a solution $\left\{w_{1}, w_{2}, s_{2}\right\}$ satisfies $0 \leq s_{2} \leq \lambda\left(w_{2}\right)$, then $w_{2}=w_{2}\left(w_{1}, s_{2}\right)$. If $s_{2}>\lambda\left(w_{2}\right)$, then $w_{2}=$ $w_{+}=w_{3}\left(w_{1}, s_{2}\right)$ and

$$
u_{+}-u_{-}=F\left(w_{1}, w_{+}, s_{2}\right)=F\left(w_{1}, w_{2}\left(w_{1}, s_{2}\right), s_{2}\right) .
$$

Thus, we can always take the $w_{2}$ in expression (4.2) for $G\left(w_{1}, s_{2}\right)$ to be $w_{2}\left(w_{1}, s_{2}\right)$. Therefore, we can always assume $0 \leq s_{2} \leq \lambda\left(w_{2}\right)$ in $G\left(w_{1}, s_{2}\right)$ since $s \leq \lambda\left(w_{2}\left(w_{1}, s\right)\right)$ due to the concavity of $p(w)$ in the region $w \geq \beta$. Similar things can be said for $J\left(w_{2}, s_{2}\right)$. 
Lemma 4.2. (i) If $0 \leq s_{2}$, then

$$
\frac{\partial G}{\partial w_{1}}\left(w_{1}, s_{2}\right) \geq \lambda\left(w_{1}\right)+s_{2}
$$

(ii) If $0 \leq s_{2} \leq \lambda\left(w_{2}\right)$, then

$$
\frac{\partial G}{\partial s_{2}}\left(w_{1}, s_{2}\right) \leq 0
$$

The equality holds only if $s_{2}=\lambda\left(w_{2}\right)$.

Proof. In this proof, we treat $w_{1}, s_{2}$ as variables. A straightforward computation based on (4.3) shows that

$$
\begin{gathered}
\frac{\partial s_{1}}{\partial w_{1}}=\frac{\lambda^{2}\left(w_{1}\right)-s_{1}^{2}}{2 s_{1}\left(w_{1}-w_{-}\right)}, \\
\frac{\partial w_{2}}{\partial w_{1}}=\frac{\lambda^{2}\left(w_{1}\right)-s_{2}^{2}}{\lambda^{2}\left(w_{2}\right)-s_{2}^{2}}, \\
\frac{\partial w_{2}}{\partial s_{2}}=\frac{2 s_{2}\left(w_{2}-w_{1}\right)}{\lambda^{2}\left(w_{2}\right)-s_{2}^{2}}, \\
\frac{\partial s_{3}}{\partial w_{2}}=\frac{s_{3}^{2}-\lambda^{2}\left(w_{2}\right)}{2 s_{3}\left(w_{+}-w_{2}\right)} .
\end{gathered}
$$

With these preparations, we can compute $\frac{\partial G}{\partial w_{1}}$ as follows:

$$
\begin{aligned}
\frac{\partial G}{\partial w_{1}}= & H\left(w_{-}-w_{1}\right)\left[-\left(w_{1}-w_{-}\right) \frac{\partial s_{1}}{\partial w_{1}}-s_{1}\right]+H\left(w_{1}-w_{-}+\right) \lambda\left(w_{1}\right)+s_{2} \\
& +\frac{\partial w_{2}}{\partial w_{1}}\left[-s_{2}-H\left(w_{2}-w_{+}\right)\left(\left(w_{+}-w_{2}\right) \frac{\partial s_{3}}{\partial w_{2}}-s_{3}\right)\right. \\
& \left.+H\left(w_{+}-w_{2}+\right) \lambda\left(w_{2}\right)\right] \\
= & H\left(w_{-}-w_{1}\right) \frac{\lambda^{2}\left(w_{1}\right)-2 s_{1} s_{2}+s_{1}^{2}}{-2 s_{1}}+H\left(w_{1}-w_{-}+\right)\left(\lambda\left(w_{1}\right)+s_{2}\right) \\
& +\frac{\lambda^{2}\left(w_{1}\right)-s_{2}^{2}}{\lambda^{2}\left(w_{2}\right)-s_{2}^{2}}\left[H\left(w_{2}-w_{+}\right) \frac{\lambda^{2}\left(w_{2}\right)-2 s_{2} s_{3}+s_{3}^{2}}{2 s_{3}}\right. \\
& \left.+H\left(w_{+}-w_{2}+\right)\left(\lambda\left(w_{2}\right)-s_{2}\right)\right] .
\end{aligned}
$$

Applying the inequality $\lambda^{2}\left(w_{1}\right)+s_{1}^{2} \geq-2 s_{1} \lambda\left(w_{1}\right), s_{1}<0$, we can prove that

$$
H\left(w_{-}-w_{1}\right) \frac{\lambda^{2}\left(w_{1}\right)-2 s_{1} s_{2}+s_{1}^{2}}{-2 s_{1}}+H\left(w_{1}-w_{-}+\right)\left(\lambda\left(w_{1}\right)+s_{2}\right) \geq \lambda\left(w_{1}\right)+s_{2}
$$

Recalling constraints (4.4), we can see easily that the sum of the last two terms on the right-hand side of (4.15) is positive. This completes the proof of (i). 
(ii) After a computation similar to those in (i), we obtain

$$
\begin{aligned}
\frac{\partial G}{\partial s_{2}}=\frac{w_{1}-w_{2}}{\lambda\left(w_{2}\right)^{2}-s_{2}^{2}}\left[H\left(w_{2}-w_{+}\right)\right. & \frac{1}{s_{3}}\left(s_{3}-s_{2}\right)\left(\lambda\left(w_{2}\right)^{2}-s_{2} s_{3}\right) \\
+ & \left.H\left(w_{+}-w_{2}+\right)\left(\lambda\left(w_{2}\right)-s_{2}\right)^{2}\right] .
\end{aligned}
$$

An analysis similar to that for (4.15) yields our result.

Lemma 4.3. (i) If $s_{2} \leq 0$, then

$$
\frac{\partial J}{\partial w_{2}}\left(w_{2}, s_{2}\right)>\lambda\left(w_{2}\right)-s_{2}
$$

(ii) If $0 \geq s_{2} \geq \lambda\left(w_{1}\right)$, then

$$
\frac{\partial J}{\partial s_{2}}\left(w_{2}, s_{2}\right) \leq 0
$$

The equality holds only if $s_{2}=-\lambda\left(w_{1}\right)$.

Proof. The proof is almost the same as that of Lemma 4.2. For completeness, we list some of the intermediate results in the following:

$$
\begin{aligned}
\frac{\partial J}{\partial w_{1}}= & \frac{\lambda^{2}\left(w_{2}\right)-s_{2}^{2}}{\lambda^{2}\left(w_{1}\right)-s_{2}^{2}}\left[H\left(w_{-}-w_{1}\right) \frac{\lambda^{2}\left(w_{1}\right)-2 s_{1} s_{2}+s_{1}^{2}}{-2 s_{1}}\right. \\
& \left.+H\left(w_{2}-w_{+}\right) \frac{\lambda^{2}\left(w_{2}\right)-2 s_{2} s_{3}+s_{3}^{2}}{2 s_{3}}+H\left(w_{+}-w_{-}+\right)\left(\lambda\left(w_{1}\right)+s_{2}\right)\right] \\
= & \frac{w_{1}-w_{2}}{\lambda\left(w_{1}\right)^{2}-s_{2}^{2}}\left[H\left(w_{-}\right)-w_{1}\right), \frac{\partial J}{s_{1}}\left(s_{1}-s_{2}\right)\left(\lambda\left(w_{1}\right)^{2}-s_{2} s_{1}\right) \\
& \left.+H\left(w_{1}-w_{-}+\right)\left(\lambda\left(w_{1}\right)+s_{2}\right)^{2}\right] .
\end{aligned}
$$

Lemma 4.4. (i) (1.1) has at most one solution $\left\{w_{1}, w_{2}, s_{2}\right\}$ with $s_{2} \geq 0$.

(ii) (1.1) has at most one solution $\left\{w_{1}, w_{2}, s_{2}\right\}$ with $s_{2} \leq 0$.

Proof. (i) We claim that (1.1) can have at most one solution $\left\{w_{1}, w_{2}, s_{2}\right\}$ with

$$
0 \leq s_{2} \leq \lambda\left(w_{2}\right) \text {. }
$$

Indeed, if we have two solutions of (1.1), $\left\{w_{1}, w_{2}, s_{2}\right\}$ and $\left\{\bar{w}_{1}, \bar{w}_{2}, \bar{s}_{2}\right\}$, with (4.18) and

$$
0 \leq \bar{s}_{2} \leq \lambda\left(\bar{w}_{2}\right)
$$

and $\left(w_{1}, s_{2}\right) \neq\left(\bar{w}_{1}, \bar{s}_{2}\right)$, then we have, by Lemma 4.1 ,

$$
G\left(w_{1}, s_{2}\right)=G\left(\bar{w}_{1}, \bar{s}_{2}\right)=u_{+}-u_{-} .
$$

(4.20) shows, with help from Lemma 4.2(i), that $w_{1} \neq \bar{w}_{1}$. Without loss of generality, we assume that

$$
\bar{w}_{1}>w_{1}
$$

Then, by Theorem 3.6,

$$
\bar{s}_{2}<s_{2} \text {. }
$$


Consider the difference

$$
\begin{aligned}
G\left(\bar{w}_{1}, \bar{s}_{2}\right)-G\left(w_{1}, s_{2}\right) & \\
= & G\left(\bar{w}_{1}, \bar{s}_{2}\right)-G\left(\bar{w}_{1}, s_{2}\right)+G\left(\bar{w}_{1}, s_{2}\right)-G\left(w_{1}, s_{2}\right) \\
= & \left.\frac{\partial G}{\partial w_{1}^{*}}\left(w_{1}^{*}, s_{2}\right)\right|_{w_{1}^{*}=w_{0} \in\left(w_{1}, \bar{w}_{1}\right)}\left(\bar{w}_{1}-w_{1}\right) \\
& \quad+\left.\frac{\partial G}{\partial s_{2}^{*}}\left(\bar{w}_{1}, s_{2}^{*}\right)\right|_{s_{2}^{*}=s_{0} \in\left(\bar{s}_{2}, s_{2}\right)}\left(\bar{s}_{2}-s_{2}\right) .
\end{aligned}
$$

Applying Remark 4.1, (4.21), (4.22), and Lemma 4.2, we find that

$$
G\left(\bar{w}_{1}, \bar{s}_{2}\right)>G\left(w_{1}, s_{2}\right)=u_{+}-u_{-},
$$

which contradicts (4.20). Thus, our claim holds.

Now, we claim that $(1.1)$ has at most one solution $\left\{w_{1}, w_{2}, s_{2}\right\}$ with

$$
s_{2}>\lambda\left(w_{2}\right) \text {. }
$$

In this case, $w_{2}=w_{+}$, and hence it is necessary, in view of Lemma 4.1, that

$$
F\left(w_{1}, w_{+}, s_{2}\right)=u_{+}-u_{-}
$$

After some computation, we obtain

$$
\begin{aligned}
\frac{d F}{d w_{1}}= & H\left(w_{-}-w_{1}\right) \frac{1}{2}\left(s_{2}-s_{1}\right)\left(1-\frac{\lambda\left(w_{1}\right)^{2}}{s_{2} s_{1}}\right) \\
& +H\left(w_{1}-w_{-}+\right) \frac{\left(\lambda\left(w_{1}\right)+s_{2}\right)^{2}}{2 s_{2}}>0
\end{aligned}
$$

if (4.25) holds. Thus, (4.26) has at most one solution $w_{1}$ and our claim is proven.

It remains to show that (1.1) cannot have a solution satisfying (4.18) and a solution satisfying (4.25) simultaneously. Suppose, for contradiction, that (1.1) has a solution $\left\{w_{1}, w_{2}, s_{2}\right\}$ satisfying (4.18) and a solution $\left\{\bar{w}_{1}, \bar{w}_{2}, \bar{s}_{2}\right\}$ satisfying (4.25) and hence $\bar{w}_{2}=w_{+}$.

We define

$$
\begin{aligned}
& w_{2}\left(w_{1}, s\right):=\max \left\{w \geq \beta \mid p(w)=p\left(w_{1}\right)-s^{2}\left(w-w_{1}\right)\right\} \\
& w_{3}\left(w_{1}, s\right):=\min \left\{w \geq \beta \mid p(w)=p\left(w_{1}\right)-s^{2}\left(w-w_{1}\right)\right\}
\end{aligned}
$$

Case 1. $w_{1}>\bar{w}_{1}$. In this case, by Theorem 3.6,

$$
\bar{s}_{2}>s_{2} \text {. }
$$

If, further, $w_{3}\left(w_{1}, s_{2}\right)>w_{+}$, then an inspection of the graph of $p(w)$ will tell us that $s_{3}<s_{2}$, which is unacceptable by (4.4). If, on the other hand, $w_{3}\left(w_{1}, s_{2}\right) \leq w_{+}$, then, by Lemma 4.2,

$$
G\left(w_{1}, s_{2}\right)>G\left(w_{1}, \bar{s}_{2}\right)>G\left(\bar{w}_{1}, \bar{s}_{2}\right)=F\left(\bar{w}_{1}, \bar{w}_{+}, \bar{s}_{2}\right)=u_{+}-u_{-} .
$$

(4.30) implies, by virtue of Lemma 4.1 , that $\left\{w_{1}, w_{2}, s_{2}\right\}$ is not a solution of (1.1), which contradicts our assumption. 
Case 2. $w_{1}<\bar{w}_{1}$. For the same reason as for Case 1 , we can assume, without loss of generality, that $w_{3}\left(w_{1}, s_{2}\right) \leq w_{+}$. A calculation of

$$
\frac{d}{d w}\left(-\frac{p(w)-p(u)}{w-u}\right)
$$

shows that $s_{2}>\bar{s}_{2}$ Then, by virtue of Lemma 4.2,

$$
G\left(w_{1}, s_{2}\right)<G\left(\bar{w}_{1}, s_{2}\right)<G\left(\bar{w}_{1}, \bar{s}_{2}\right)=F\left(\bar{w}_{1}, w_{+}, \bar{s}_{2}\right)=u_{+}-u_{-} .
$$

Thus, $\left\{w_{1}, w_{2}, s_{2}\right\}$ is not a solution of (1.1). We again get a contradiction.

Case 3. $w_{1}=\bar{w}_{1}$. Theorem 3.5 states that, in this case, $w_{3}\left(w_{1}, s_{2}\right)>w_{+}$and hence $\left\{w_{1}, w_{2}, s_{2}\right\}$ is not a solution of (1.1).

Our discussion of the above three cases proves assertion (i).

(ii) The proof of (ii) is similar to that of (i).

Theorem 4.5. (1.1) has a unique centered wave solution which is admissible according to the viscosity-capillarity travelling wave criterion.

Proof. The existence part of our theorem is given by Theorem 2.1.

To prove the uniqueness of the solution of (1.1), it suffices to show that cases (i) and (ii) in Lemma 4.4 are mutually exclusive. Assume the contrary, i.e., there are solutions of (1.1), $\left\{w_{1}, w_{2}, s_{2}\right\}$ and $\left\{\bar{w}_{1}, \bar{w}_{2}, \bar{s}_{2}\right\}$, with $s_{2} \geq 0$ and $\bar{s}_{2}<0$. Then

$$
G\left(w_{1}, s_{2}\right)=J\left(\bar{w}_{2}, \bar{s}_{2}\right)=u_{+}-u_{-} .
$$

By Lemma 3.6,

$$
w_{1} \leq m, \quad \bar{w}_{2}>M,
$$

where $m$ and $M$ are the Maxwell constants defined by (3.4). Similar to what we did in the proof of Theorem 4.4, we can show that

$$
u_{+}-u_{-}=G\left(w_{1}, s_{2}\right) \leq G(m, 0)=J(M, 0)<J\left(\bar{w}_{2}, \bar{s}_{2}\right)=u_{+}-u_{-},
$$

which is impossible.

The uniqueness of the solution $\left\{w_{1}, w_{2}, s_{2}\right\}$ of (1.1) enables us to think of $w_{1}, w_{2}, s_{2}$ as functions of $u_{ \pm}, w_{ \pm}$:

$$
\begin{gathered}
w_{k}=w_{k}\left(u_{-}, u_{+}, w_{-}, w_{+}\right), \quad k=1,2, \\
s_{2}=s_{2}\left(u_{-}, u_{+}, w_{-}, w_{+}\right) .
\end{gathered}
$$

For convenience, we shall denote the solution of (1.1) by $\left\{w_{1}, w_{2}, s_{2}, u_{ \pm}, w_{ \pm}\right\}$ in the rest of this paper.

Lemma 4.6. (i) Let $\left\{w_{1}, w_{2}, s_{2}, u_{ \pm}, w_{ \pm}\right\}$and $\left\{\bar{w}_{1}, \bar{w}_{2}, \bar{s}_{2}, \bar{u}_{ \pm}, \bar{w}_{ \pm}\right\}$be two solutions of $(1.1 \mathrm{a}, \mathrm{b})$ with $s_{2} \geq 0, \bar{s}_{2} \geq 0$. For any $\varepsilon>0$, there exists $a \delta>0$ such that if

$$
\left|u_{-}-\bar{u}_{-}\right|+\left|u_{+}-\bar{u}_{+}\right|+\left|w_{-}-\bar{w}_{-}\right|+\left|w_{+}-\bar{w}_{+}\right|<\delta
$$

then $\left|w_{k}-\bar{w}_{k}\right|<\varepsilon, k=1,2,\left|s_{2}-\bar{s}_{2}\right|<\varepsilon$.

(ii) Let $\left\{w_{1}, w_{2}, s_{2}, u_{ \pm}, w_{ \pm}\right\}$and $\left\{\bar{w}_{1}, \bar{w}_{2}, \bar{s}_{2}, \bar{u}_{ \pm}, \bar{w}_{ \pm}\right\}$be two solutions of $(1.1 \mathrm{a}, \mathrm{b})$ with $s_{2} \leq 0, \bar{s}_{2} \leq 0$. For any $\varepsilon>0$, there exists $a \delta>0$ such that if (4.34) is satisfied then $\left|w_{i}-\bar{w}_{i}\right|<\varepsilon, k=1,2,\left|s_{2}-\bar{s}_{2}\right|<\varepsilon$.

Proof. We define an auxiliary function as follows:

$$
K\left(w_{1}, s_{2}, u_{ \pm}, w_{ \pm}\right):=G\left(w_{1}, s_{2}\right)-u_{+}-u_{-} .
$$


By Lemma 4.1, a necessary condition for $\left\{w_{1}, w_{2}, s_{2}, u_{ \pm}, w_{ \pm}\right\}$to be a solution of $(1.1 \mathrm{a}, \mathrm{b})$ is

$$
K\left(w_{1}, s_{2}, u_{ \pm}, w_{ \pm}\right)=0
$$

We also have

$$
K\left(\bar{w}_{1}, \bar{s}_{2}, \bar{u}_{ \pm}, \bar{w}_{ \pm}\right)=0 .
$$

Without loss of generality, we assume

$$
\bar{w}_{1} \leq w_{1} .
$$

Case 1.

$$
\bar{s}_{2} \geq s_{2}
$$

Consider the following equation:

$$
\begin{aligned}
& K\left(w_{1}, \bar{s}_{2}, \bar{u}_{ \pm}, \bar{w}_{ \pm}\right)-K\left(w_{1}, \bar{s}_{2}, u_{ \pm}, w_{ \pm}\right) \\
&= K\left(w_{1}, s_{2}, u_{ \pm}, w_{ \pm}\right)-K\left(w_{1}, \bar{s}_{2}, u_{ \pm}, w_{ \pm}\right) \\
&+K\left(w_{1}, \bar{s}_{2}, \bar{u}_{ \pm}, \bar{w}_{ \pm}\right)-K\left(\bar{w}_{1}, \bar{s}_{2}, \bar{u}_{ \pm}, \bar{w}_{ \pm}\right) .
\end{aligned}
$$

Note that all the variables in (4.39) are in the domain of definition (4.4) of $G\left(w_{1}, s_{2}\right)$ and hence that of $K(\cdots)$. Since the function $K(\cdots)$ is continuous in $u_{ \pm}$and $w_{ \pm}$, for any given $\varepsilon^{\prime}>0$, there is $\delta_{1}>0$ such that if (4.34) is satisfied with $\delta=\delta_{1}$ then

$$
\left|K\left(w_{1}, \bar{s}_{2}, u_{ \pm}, w_{ \pm}\right)-K\left(w_{1}, \bar{s}_{2}, \bar{u}_{ \pm}, \bar{w}_{ \pm}\right)\right|<\varepsilon^{\prime} .
$$

Then we have, by Remark 4.1, Lemma 4.2, and (4.37), as well as (4.38), that

$$
\begin{array}{r}
K\left(w_{1}, s_{2}, u_{ \pm}, w_{ \pm}\right)-K\left(w_{1}, \bar{s}_{2}, u_{ \pm}, w_{ \pm}\right) \\
=\left.\frac{\partial G}{\partial s_{2}^{*}}\left(w_{1}, s_{2}^{*}\right)\right|_{s_{2}^{*} \in\left(s_{2}, \bar{s}_{2}\right)}\left(s_{2}-\bar{s}_{2}\right)>0
\end{array}
$$

and

$$
\begin{aligned}
& K\left(w_{1}, \bar{s}_{2}, \bar{u}_{ \pm}, \bar{w}_{ \pm}\right)-K\left(\bar{w}_{1}, \bar{s}_{2}, \bar{u}_{ \pm}, \bar{w}_{ \pm}\right) \\
& \quad=\left.\frac{\partial G}{\partial w_{1}^{*}}\left(w_{1}^{*}, \bar{s}_{2}\right)\right|_{w_{1}^{*} \in\left(\bar{w}_{1}, w_{1}\right)}\left(w_{1}-\bar{w}_{1}\right)>0 .
\end{aligned}
$$

Thus, (4.39) and (4.40) imply

$$
\begin{gathered}
0<K\left(w_{1}, s_{2}, u_{ \pm}, w_{ \pm}\right)-K\left(w_{1}, \bar{s}_{2}, u_{ \pm}, w_{ \pm}\right)<\varepsilon^{\prime}, \\
\left|\frac{\partial G}{\partial w_{1}^{*}}\left(w_{1}^{*}, \bar{s}_{2}\right)\right|_{w_{1}^{*} \in\left(\bar{w}_{1}, w_{1}\right)}\left(w_{1}-\bar{w}_{1}\right) \mid<\varepsilon^{\prime}
\end{gathered}
$$

Lemma 3.1, Lemma 4.2, and (4.43b) yield

$$
\left|w_{1}-\bar{w}_{1}\right|<\varepsilon^{\prime} / \lambda(m) \text {. }
$$

We claim that $\bar{s}_{2}<s_{2}+\varepsilon$ if (4.34) is satisfied for some $\delta>0$. To this end, we assume the contrary, i.e., $\bar{s}_{2} \geq s_{2}+\varepsilon$. We rewrite (4.43) as

$$
\begin{aligned}
\varepsilon^{\prime}> & K\left(w_{1}, s_{2}, u_{ \pm}, w_{ \pm}\right)-K\left(w_{1}, \bar{s}_{2}, u_{ \pm}, w_{ \pm}\right) \\
= & K\left(w_{1}, s_{2}, u_{ \pm}, w_{ \pm}\right)-K\left(w_{1}, s_{2}+\varepsilon / 2, u_{ \pm}, w_{ \pm}\right) \\
& +K\left(w_{1}, s_{2}+\varepsilon / 2, u_{ \pm}, w_{ \pm}\right)-K\left(w_{1}, s_{2}+\varepsilon, u_{ \pm}, w_{ \pm}\right) \\
& +K\left(w_{1}, s_{2}+\varepsilon, u_{ \pm}, w_{ \pm}\right)-K\left(w_{1}, \bar{s}_{2}, u_{ \pm}, w_{ \pm}\right)
\end{aligned}
$$


In the same way as we proceeded to get (4.43), we can derive from (4.45) that

$$
\begin{aligned}
\varepsilon^{\prime} & >K\left(w_{1}, s_{2}+\varepsilon / 2, u_{ \pm}, w_{ \pm}\right)-K\left(w_{1}, s_{2}+\varepsilon, u_{ \pm}, w_{ \pm}\right) \\
& =\left.\frac{\partial G}{\partial s_{2}^{*}}\left(w_{1}, s_{2}^{*}\right)\right|_{s_{2}^{*} \in\left(s_{2}+\varepsilon / 2, s_{2}+\varepsilon\right)} \varepsilon / 2 .
\end{aligned}
$$

From the definition of $w_{2}\left(w_{1}, s\right),(4.28 \mathrm{a})$, we can see that

$$
s_{2} \leq \lambda\left(w_{2}\left(w_{1}, s_{2}\right)\right)
$$

By the concavity of $p(w)$ in the region $w>\beta$, we have

$$
s<\lambda\left(w_{2}\left(w_{1}, s\right)\right) \text { for } s \in\left[s_{2},+\varepsilon / 2, s_{2}+\varepsilon\right] .
$$

Since $\lambda\left(w_{2}\left(w_{1}, s\right)\right)$ is continuous in $s$, there is $\mu\left(s_{2}, \varepsilon\right)>0$ such that

$$
s \leq \lambda\left(w_{2}\left(w_{1}, s\right)\right)-\mu\left(s_{2}, \varepsilon\right) .
$$

By the same reason, we can further choose $\mu\left(s_{2}, \varepsilon\right)>0$ such that

$$
s_{3}\left(w_{2}\left(w_{1}, s\right), w_{+}\right)-s \geq \mu\left(s_{2}, \varepsilon\right)>0 \quad \text { for } s \in\left[s_{2},+\varepsilon / 2, s_{2}+\varepsilon\right],
$$

where

$$
s_{3}\left(w_{2}, w_{+}\right):=-\frac{p\left(w_{2}\right)-p\left(w_{+}\right)}{w_{2}-w_{+}} .
$$

With the help of (4.47), (4.48), and (4.4b), we can estimate (4.16) as follows:

$$
\begin{aligned}
\left|\frac{\partial G}{\partial s_{2}}\left(w_{1}, s_{2}\right)\right|= & \mid \frac{w_{1}-w_{2}}{\lambda^{2}\left(w_{2}\right)-s_{2}^{2}}\left[H\left(w_{2}-w_{+}\right) \frac{1}{s_{3}}\left(s_{3}-s_{2}\right)\left(\lambda^{2}\left(w_{2}\right)-s_{2} s_{3}\right)\right. \\
& \left.+H\left(w_{+}-w_{2}+\right)\left(\lambda\left(w_{2}\right)-s_{2}\right)^{2}\right] \mid \\
> & (\beta-\alpha)\left[H\left(w_{2}-w_{+}\right) \frac{\left(s_{3}-s_{2}\right)}{2 \lambda\left(w_{2}\right)}+H\left(w_{+}-w_{2}+\right) \frac{\lambda\left(w_{2}\right)-s_{2}}{2 \lambda\left(w_{2}\right)}\right] \\
> & C\left(w_{1}, s_{2}, \varepsilon\right)>0 .
\end{aligned}
$$

Recalling (4.46), we obtain

$$
\varepsilon^{\prime}>C\left(w_{1}, s_{2}, \varepsilon\right) \varepsilon / 2 \text {. }
$$

However, $\varepsilon^{\prime}$ can be chosen independently of $\varepsilon$. For example, we can choose

$$
\varepsilon^{\prime}<C\left(w_{1}, s_{2}, \varepsilon\right) \varepsilon / 2 \text {. }
$$

Then there exists a $\delta_{2}>0$ such that if (4.34) is satisfied with $\delta=\delta_{1}$, then (4.46) and hence (4.49) hold, which contradicts our choice of $\varepsilon^{\prime}$. This contradiction proves our claim.

The fact that $\left|w_{2}-\bar{w}_{2}\right|<\varepsilon$ if (4.34) holds for some $\delta=\delta_{3}>0$ is a consequence of the fact that $w_{2}$ depends on $w_{1}$ and $s_{2}$ continuously. Now, we choose

$$
\varepsilon^{\prime} \leq \min \left(\varepsilon \lambda(m), C\left(w_{1}, s_{2}, \varepsilon\right) \varepsilon / 2\right)
$$

and then $\delta=\min \left(\delta_{1}, \delta_{2}, \delta_{3}\right)$ is the one needed by our assertion.

Case 2. $0 \leq \bar{s}_{2}<s_{2}$. In this case, by Theorem 3.5, $w_{1} \rightarrow w_{3}\left(w_{1}, s_{2}\right)$ rather than $w_{1} \rightarrow w_{2}\left(w_{1}, s_{2}\right)$ is a connection. An inspection of the graph of $p(w)$ tells us that

$$
\bar{w}_{+} \geq w_{3}\left(\bar{w}_{1}, \bar{s}_{2}\right)=: \bar{w}_{3}>w_{3}:=w_{3}\left(w_{1}, s_{2}\right)=w_{+}
$$


or

$$
\left|w_{3}-\bar{w}_{3}\right|<\left|w_{+}-\bar{w}_{+}\right|
$$

From (4.3), we have

$$
s_{2}^{2}\left(w_{3}-w_{1}\right)=-p\left(w_{3}\right)+p\left(w_{1}\right)
$$

and

$$
\bar{s}_{2}^{2}\left(\bar{w}_{3}-\bar{w}_{1}\right)=-p\left(\bar{w}_{3}\right)+p\left(\bar{w}_{1}\right)
$$

In the following part of our proof, we shall assume, without loss of generality, that $\delta \leq 1$. After subtracting (4.52a) from (4.52b) and some manipulations on the difference, we obtain

$$
\begin{aligned}
& \left(\lambda^{2}\left(w_{1}^{*}\right)-\bar{s}_{2}^{2}\right)\left(w_{1}-\bar{w}_{1}\right)+\left(w_{3}-w_{1}\right)\left(s_{2}^{2}-\bar{s}_{2}^{2}\right) \\
& \quad=\left(p^{\prime}\left(w_{3}^{*}\right)-\bar{s}_{2}^{2}\right)\left(w_{3}-\bar{w}_{3}\right) \\
& \quad \leq\left(\max _{w \in\left[w_{+}, \bar{w}_{+}\right]}\left|p^{\prime}(w)\right|+s_{2}^{2}\right)\left|w_{3}-\bar{w}_{3}\right| \\
& \quad \leq\left(\max _{w \in\left[w_{+}, w_{+}+1\right]}\left|p^{\prime}(w)\right|+s_{2}^{2}\right)\left|w_{+}-\bar{w}_{+}\right| \\
& \quad:=C_{2}\left(w_{+}, s_{2}\right)\left|w_{+}-\bar{w}_{+}\right|
\end{aligned}
$$

where $w_{1}^{*} \in\left(\bar{w}_{1}, w_{1}\right)$ and $w_{3}^{*} \in\left(w_{3}, \bar{w}_{3}\right)$. Each term on the left-hand side of (4.53) is nonnegative; hence

$$
0 \leq w_{1}-\bar{w}_{1} \leq \frac{C_{2}\left(w_{+}, s_{2}\right)}{\lambda^{2}\left(w_{1}\right)-s_{2}^{2}}\left|w_{+}-\bar{w}_{+}\right|
$$

and

$$
0 \leq s_{2}-\bar{s}_{2} \leq \frac{C_{2}\left(w_{+}, s_{2}\right)}{2 s_{2}(\beta-\alpha)}\left|w_{+}-\bar{w}_{+}\right|
$$

Thus, our assertion holds for this case.

(ii) The proof for (ii) is almost similar to that of (i) except that we have to work on the auxiliary function

$$
L\left(w_{2}, s_{2}, u_{ \pm}, w_{ \pm}\right):=J\left(w_{2}, s_{2}\right)-u_{+}-u_{-}
$$

instead of $K\left(w_{1}, s_{2}, u_{ \pm}, w_{ \pm}\right)$.

Theorem 4.7. $w_{k}\left(u_{-}, u_{+}, w_{-}, w_{+}\right), k=1,2$, and $s_{2}\left(u_{-}, u_{+}, w_{-}, w_{+}\right)$are continuous functions for $w_{-} \leq \alpha<\beta \leq w_{+}$.

Proof. Let $\left\{w_{1}, w_{2}, s_{2}, u_{ \pm}, w_{ \pm}\right\}$and $\left\{\bar{w}_{1}, \bar{w}_{2}, \bar{s}_{2}, \bar{u}_{ \pm}, \bar{w}_{ \pm}\right\}$be two solutions of $(1.1 \mathrm{a}, \mathrm{b})$. Our assertion is equivalent to: for any $\varepsilon>0$, there exists a $\delta>0$ such that if

$$
\left|u_{-}-\bar{u}_{-}\right|+\left|u_{+}-\bar{u}_{+}\right|+\left|w_{-}-\bar{w}_{-}\right|+\left|w_{+}-\bar{w}_{+}\right|<\delta,
$$

then $\left|w_{k}-\bar{w}_{k}\right|<\varepsilon, k=1,2,\left|s_{2}-\bar{s}_{2}\right|<\varepsilon$. To this end, since we have Lemma 4.6 , it suffices to show our assertion for the case when $s_{2} \geq 0$ and $\bar{s}_{2}<0$. By Lemma 4.1, we have

$$
K\left(w_{1}, s_{2}, u_{ \pm}, w_{ \pm}\right)=0
$$




$$
L\left(\bar{w}_{2}, \bar{s}_{2}, \bar{u}_{ \pm}, \bar{w}_{ \pm}\right)=0 .
$$

It follows from above that

$$
\begin{aligned}
& K\left(m, 0, u_{ \pm}, w_{ \pm}\right)-K\left(m, 0, \bar{u}_{ \pm}, \bar{w}_{ \pm}\right) \\
&= K\left(m, 0, u_{ \pm}, w_{ \pm}\right)-L\left(M, 0, \bar{u}_{ \pm}, \bar{w}_{ \pm}\right) \\
&= K\left(m, 0, u_{ \pm}, w_{ \pm}\right)-K\left(w_{1}, s_{2}, u_{ \pm}, w_{ \pm}\right) \\
&+L\left(\bar{w}_{2}, \bar{s}_{2}, \bar{u}_{ \pm}, \bar{w}_{ \pm}\right)-L\left(M, 0, \bar{u}_{ \pm}, \bar{w}_{ \pm}\right) .
\end{aligned}
$$

It is clear from Lemma 4.2 that the sum of the first two terms on the right-hand side of (4.58) and that of the last two terms are nonnegative. Thus

$$
\begin{aligned}
0 & \leq K\left(m, 0, u_{ \pm}, w_{ \pm}\right)-K\left(w_{1}, s_{2}, u_{ \pm}, w_{ \pm}\right) \\
& \leq\left|K\left(m, 0, u_{ \pm}, w_{ \pm}\right)-K\left(m, 0, \bar{u}_{ \pm}, \bar{w}_{ \pm}\right)\right|, \\
0 & \leq L\left(\bar{w}_{2}, \bar{s}_{2}, \bar{u}_{ \pm}, \bar{w}_{ \pm}\right)-L\left(M, 0, \bar{u}_{ \pm}, \bar{w}_{ \pm}\right) \\
& \leq\left|K\left(m, 0, u_{ \pm}, w_{ \pm}\right)-K\left(m, 0, \bar{u}_{ \pm}, \bar{w}_{ \pm}\right)\right| .
\end{aligned}
$$

Applying Lemma 4.6 to (4.59), we find that for any $\varepsilon>0$, there is a $\delta>0$ such that if (4.56) is satisfied then

$$
\begin{aligned}
\left|w_{1}-m\right|<\varepsilon / 2, & & \left|w_{2}-M\right|<\varepsilon / 2, \\
\left|s_{2}\right|<\varepsilon / 2, & & \left|\bar{s}_{2}\right|<\varepsilon / 2, \\
\left|\bar{w}_{1}-m\right|<\varepsilon / 2, & & \left|\bar{w}_{2}-M\right|<\varepsilon / 2 .
\end{aligned}
$$

These inequalities imply our theorem.

Theorem 4.8. Let $(u(\xi), w(\xi))$ be the solution of (1.1). For any $\varepsilon>0$ and $\gamma>0$, there is a $\delta>0$ such that if

$$
\left|u_{-}-\bar{u}_{-}\right|+\left|u_{+}-\bar{u}_{+}\right|+\left|w_{-}-\bar{w}_{-}\right|+\left|w_{+}-\bar{w}_{+}\right|<\delta
$$

then

$$
\operatorname{meas}\{\xi \in \mathbb{R}|| u(\xi)-\bar{u}(\xi)|+| w(\xi)-\bar{w}(\xi) \mid \geq \varepsilon\}<\gamma,
$$

where $(\bar{u}(\xi), \bar{w}(\xi))$ is the solution of $(1.1 \mathrm{a}, \mathrm{b})$ with Riemann initial values $\left(\bar{u}_{-}, \bar{w}_{-}\right)$and $\left(\bar{u}_{+}, \bar{w}_{+}\right)$.

Proof. Solutions $(u(\xi), w(\xi))$ and $(\bar{u}(\xi), \bar{w}(\xi))$ can be written as $\left\{w_{1}, w_{2}\right.$, $\left.s_{2}, u_{ \pm}, w_{ \pm}\right\}$and $\left\{\bar{w}_{1}, \bar{w}_{2}, \bar{s}_{2}, \bar{u}_{ \pm}, \bar{w}_{ \pm}\right\}$respectively. We know from Theorem 4.7 that $w_{1}, w_{2}, s_{1}, s_{2}, s_{3}$ are continuous functions of $u_{ \pm}, w_{ \pm}$. Thus there is a $1 \geq \delta_{0}>0$ such that

$$
\sum_{k=0}^{3}\left(\left|u_{k}-\bar{u}_{k}\right|+\left|w_{k}-\bar{w}_{k}\right|\right)+\max _{w \in\left[w_{-}-1, w_{+}+1\right]}\left|\int_{w}^{w+1} \lambda(\eta) d \eta\right|<\varepsilon
$$

if $(4.60)$ is satisfied with $\delta=\delta_{0}$, where $\left(u_{0}, w_{0}\right):=\left(u_{-}, w_{-}\right),\left(u_{3}, w_{3}\right):=$ $\left(u_{+}, w_{+}\right)$.

We define

$$
\begin{array}{r}
A_{\varepsilon}:=\{\xi \in \mathbb{R} \mid w(\xi) \leq \alpha \text { or } \bar{w}(\xi) \leq \alpha, \text { such that } \\
|u(\xi)-\bar{u}(\xi)|+|w(\xi)-\bar{w}(\xi)| \geq \varepsilon\}, \\
B_{\varepsilon}:=\{\xi \in \mathbb{R} \mid w(\xi) \geq \alpha \text { or } \bar{w}(\xi) \geq \alpha, \text { such that } \\
|u(\xi)-\bar{u}(\xi)|+|w(\xi)-\bar{w}(\xi)| \geq \varepsilon\} .
\end{array}
$$


The solution $(u(\xi), w(\xi))$ consists of at most two continuous pieces in the region $\{\xi \in \mathbb{R} \mid w(\xi) \leq \alpha\}$. From the results in $\S 2$, we can express $\left\{w_{1}, w_{2}, s_{2}\right.$, $\left.u_{ \pm}, w_{ \pm}\right\}$as follows: If $w_{1}<w_{-}$, then

$$
(u(\xi), w(\xi))= \begin{cases}\left(u_{-}, w_{-}\right) & \text {for } \xi \in\left(-\infty, s_{1}\right), \\ \left(u_{1}, w_{1}\right) & \text { for } \xi \in\left(s_{1}, s_{2}\right)\end{cases}
$$

If $w_{1} \geq w_{-}$, then

$$
(u(\xi), w(\xi))=\left\{\begin{array}{c}
\left(u_{-}, w_{-}\right) \text {for } \xi \in\left(-\infty,-\lambda\left(w_{-}\right),\right. \\
\left(u_{1}, w_{1}\right) \text { for } \xi \in\left(-\lambda\left(w_{1}\right), s_{2}\right), \\
\text { for } \xi \in\left(-\lambda\left(w_{-}\right),-\lambda\left(w_{1}\right)\right),(u(\xi), w(\xi)) \text { is determined } \\
\quad \text { by } \xi^{2}=-p^{\prime}(w(\xi)) \text { and } u(\xi)=u_{-}+\int_{w_{-}}^{w(\xi)} \lambda(\eta) d \eta
\end{array}\right.
$$

In the rest of our proof, we assume that $\delta \leq \delta_{0}$ and (4.60) holds.

Case 1. $w_{1}<w_{-}$and $\bar{w}_{1}<\bar{w}_{-}$. In this case,

$$
A_{\varepsilon} \subset\left(\min \left(s_{1}, \bar{s}_{1}\right), \max \left(s_{1}, \bar{s}_{1}\right)\right) \cup\left(\min \left(s_{2}, \bar{s}_{2}\right), \max \left(s_{2}, \bar{s}_{2}\right)\right)
$$

and hence

$$
\text { meas } A_{\varepsilon} \leq\left|s_{1}-\bar{s}_{1}\right|+\left|s_{2}-\bar{s}_{2}\right| \text {. }
$$

Case 2. $w_{1} \geq w_{-}$and $\bar{w}_{1} \geq \bar{w}_{-}$. For this case,

$$
\begin{aligned}
A_{\varepsilon} \subset & \left(\min \left(-\lambda\left(w_{-}\right),-\lambda\left(\bar{w}_{-}\right)\right), \max \left(-\lambda\left(w_{-}\right),-\lambda\left(\bar{w}_{-}\right)\right)\right) \\
& \cup\left(\min \left(-\lambda\left(w_{1}\right),-\lambda\left(\bar{w}_{1}\right)\right), \max \left(-\lambda\left(w_{1}\right),-\lambda\left(\bar{w}_{1}\right)\right)\right) \\
& \cup\left(\min \left(s_{2}, \bar{s}_{2}\right), \max \left(s_{2}, \bar{s}_{2}\right)\right)
\end{aligned}
$$

and thus

$$
\text { meas } A_{\varepsilon} \leq\left|\lambda\left(w_{-}\right)-\lambda\left(\bar{w}_{-}\right)\right|+\left|\lambda\left(w_{1}\right)-\lambda\left(\bar{w}_{1}\right)\right|+\left|s_{2}-\bar{s}_{2}\right| .
$$

For both cases, since $w_{1}, s_{1}, w_{2}$ are continuous functions of $u_{ \pm}, w_{ \pm}$, we can find a $\delta_{1}>0$ such that the right-hand sides of (4.65) and (4.66) are less than $\gamma / 2$ if (4.60) holds for $\delta=\delta_{1}$.

Case 3.

$$
w_{1}<w_{-}, \quad \bar{w}_{1} \geq \bar{w}_{-} .
$$

In this case,

$$
\begin{aligned}
& A_{\varepsilon} \subset\left(\min \left(s_{1},-\lambda\left(\bar{w}_{-}\right)\right), \max \left(s_{1},-\lambda\left(\bar{w}_{-}\right)\right)\right) \\
& \cup\left(\min \left(s_{1},-\lambda\left(\bar{w}_{1}\right)\right), \max \left(s_{1},-\lambda\left(\bar{w}_{1}\right)\right)\right) \cup\left(\min \left(s_{2}, \bar{s}_{2}\right), \max \left(s_{2}, \bar{s}_{2}\right)\right)
\end{aligned}
$$

and thus

$$
\begin{aligned}
\text { meas } A_{\varepsilon} & \leq\left|s_{1}-\lambda\left(\bar{w}_{-}\right)\right|+\left|s_{1}-\lambda\left(\bar{w}_{1}\right)\right|+\left|s_{2}-\bar{s}_{2}\right| \\
& =\left|\lambda\left(w^{*}\right)-\lambda\left(\bar{w}_{-}\right)\right|+\lambda\left(w^{*}\right)-\lambda\left(\bar{w}_{1}\right)|+| s_{2}-\bar{s}_{2} \mid,
\end{aligned}
$$

where $w^{*} \in\left(w_{1}, w_{-}\right)$. There exists a $\delta_{2}>0$ such that if $(4.60)$ holds with $\delta=\delta_{2}$ and if $\left|w^{*}-\bar{w}_{-}\right|<\delta_{2}$ and $\left|w^{*}-\bar{w}_{1}\right|<\delta_{2}$, then the right-hand side of (4.68) is less than $\gamma / 2$. We can further choose $0<\delta_{3}<\delta_{2}$ such that

$$
\left|w_{-}-\bar{w}_{-}\right|+\left|w_{1}-\bar{w}_{1}\right|<\delta_{2} / 2
$$


if (4.60) is satisfied with $\delta=\delta_{3}$. Recalling (4.67), we find that

$$
\begin{aligned}
0 & \leq w_{-}-w_{1}=w_{-}-\bar{w}_{-}+\bar{w}_{-}-\bar{w}_{1}+\bar{w}_{1}-w_{1} \\
& \leq w_{-}-\bar{w}_{-}+\bar{w}_{1}-w_{1} \\
& \leq\left|w_{-}-\bar{w}_{-}\right|+\left|\bar{w}_{1}-w_{1}\right|<\delta_{2} / 2
\end{aligned}
$$

Similarly, we can prove that

$$
0 \leq \bar{w}_{1}-\bar{w}_{-} \leq\left|w_{-}-\bar{w}_{-}\right|+\left|\bar{w}_{1}-w_{1}\right|<\delta_{2} / 2 \text {. }
$$

It follows immediately from (4.60) and (4.70) that

$$
\left|w_{1}-\bar{w}_{-}\right|<\delta_{2}, \quad\left|w_{-}-\bar{w}_{1}\right|<\delta_{2}
$$

and hence

$$
\left|w^{*}-\bar{w}_{-}\right|<\delta_{2}, \quad\left|w^{*}-\bar{w}_{1}\right|<\delta_{2}
$$

for $w^{*} \in\left(w_{1}, w_{-}\right)$. Therefore, by virtue of (4.68), meas $A_{\varepsilon}<\gamma / 2$ if (4.60) holds for $\delta=\delta_{3}$.

Case 4. $w_{1} \geq w_{-}, \bar{w}_{1}<\bar{w}_{-}$. We claim that meas $A_{\varepsilon}<\gamma / 2$ if $(4.60)$ holds for some $\delta=\delta_{4}>0$. The proof is similar to that of Case 3 .

Also, we can show that meas $B_{\varepsilon}<\gamma / 2$ if (4.60) is satisfied with some $\delta=\delta_{5}$.

Now, we take $\delta=\min \left(\delta_{0}, \delta_{1}, \delta_{3}, \delta_{4}, \delta_{5}\right)$ in (4.60), Then

$$
\begin{gathered}
\operatorname{meas}\{\xi \in \mathbb{R}|| u(\xi)-\bar{u}(\xi)|+| w(\xi)-\bar{w}(\xi) \mid>\varepsilon\} \\
\quad \leq \operatorname{meas} A_{\varepsilon}+\operatorname{meas} B_{\varepsilon}<\gamma .
\end{gathered}
$$

\section{Appendix. The Proof of Lemma 2(i)}

Proof. Assume, for comtradiction, that $w\left(\xi_{0}-\right) \in(\alpha, \beta)$ for some $\xi_{0} \in \mathbb{R}$. We claim that $w(\xi+)=w\left(\xi_{0}-\right)$ for $\xi \in\left(\xi_{0}-\delta, \xi_{0}\right)$ for some $\delta>0$. Indeed, if otherwise, one of the following two cases will occur:

Case (i). $w(\xi)$ is continuous on $\left(\xi_{0}-\delta, \xi_{0}\right)$ for some $\delta>0$ and there is a sequence $\left\{\xi_{n}\right\} \subset\left(\xi_{0}-\delta, \xi_{0}\right)$ such that $\xi_{n} \rightarrow \xi_{0}-$ as $n \rightarrow \infty$ and $w\left(\xi_{n}+\right) \neq$ $w\left(\xi_{0}-\right)$.

Case (ii). There is a sequence of points of discontinuity of $(u(\xi), w(\xi))$ such that $\xi_{n} \rightarrow \xi_{0}-$ as $n \rightarrow \infty$.

Case (ii) cannot occur because $w\left(\xi_{n} \pm\right) \in(\alpha, \beta)$ for large $n$ and the RankineHugoniot conditions at $\xi=\xi_{n}$ cannot hold.

We claim that Case (i) is also impossible. Indeed, we can integrate (1.4) over $\left(\xi_{n}, \xi_{0}\right)$, to get

$$
\xi_{0} \frac{\Delta_{n} u}{\Delta_{n} w}=p^{\prime}(\theta)-\frac{1}{\Delta_{n} w} \int_{\xi_{n}+}^{\xi_{0}-}\left(u(\zeta)-u\left(\xi_{n}+\right)\right) d \zeta
$$

$$
\frac{\Delta_{n} u}{\Delta_{n} w}=-\xi_{0}-\frac{1}{\Delta_{n} w} \int_{\xi_{n}+}^{\xi_{0}-}\left[w\left(\xi_{n}+\right)-w(\zeta)\right] d \zeta,
$$

where $\Delta_{n} w:=w\left(\xi_{0}-\right)-w\left(\xi_{n}+\right)>0, \Delta_{n} u:=u\left(\xi_{0}-\right)-u\left(\xi_{n}+\right)$ and $\theta \in$ $\left(w\left(\xi_{n}+\right), w\left(\xi_{0}-\right)\right)$. It follows from Lemma 7.4.1 that

$$
\xi_{0} \lim _{n \rightarrow \infty} \frac{\Delta_{n} u}{\Delta_{n} w}=p^{\prime}\left(w\left(\xi_{0}-\right)\right), \quad \lim _{n \rightarrow \infty} \frac{\Delta_{n} u}{\Delta_{n} w}=-\xi_{0} .
$$


Then we arrive at the contradiction

$$
\left(\lim _{n \rightarrow \infty} \frac{\Delta_{n} u}{\Delta_{n} w}\right)^{2}=-p^{\prime}\left(w\left(\xi_{0}-\right)\right)<0 .
$$

It follows that there exists $\xi_{1}$ and $\xi_{2}$ which are points of discontinuity of $(u(\xi), w(\xi))$ such that $\xi_{1}<\xi_{0}<\xi_{2}$ and

$$
w(\xi)=w\left(\xi_{0}-\right) \in(\alpha, \beta) \text { for } \xi \in\left(\xi_{1}, \xi_{2}\right) .
$$

Therefore, according to the travelling wave criterion, both boundary value problems

$$
\begin{gathered}
\frac{d^{2} \hat{w}}{d \zeta^{2}}=-2 \xi_{1} \frac{d \hat{w}(\zeta)}{d \zeta}-\xi_{1}^{2}\left(\hat{w}(\zeta)-w\left(\xi_{1}+\right)\right)-\left(p(\hat{w}(\zeta))-p\left(w\left(\xi_{1}+\right)\right)\right) \\
\hat{w}(-\infty)=w\left(\xi_{1}-\right), \quad \hat{w}(+\infty)=w\left(\xi_{1}+\right) \\
\hat{w}^{\prime}( \pm \infty)=0
\end{gathered}
$$

and

$$
\begin{gathered}
\frac{d^{2} \hat{w}}{d \zeta^{2}}=-2 \xi_{2} \frac{d \hat{w}(\zeta)}{d \zeta}-\xi_{2}^{2}\left(\hat{w}(\zeta)-w\left(\xi_{2}-\right)\right)-\left(p(\hat{w}(\zeta))-p\left(w\left(\xi_{2}-\right)\right)\right) \\
\hat{w}(-\infty)=w\left(\xi_{2}-\right), \quad \hat{w}(+\infty)=w\left(\xi_{2}+\right) \\
\hat{w}^{\prime}( \pm \infty)=0
\end{gathered}
$$

have solutions. A straightforward calculation shows that the eigenvalue for the linearized (near $\left.\hat{w}(\zeta)=w\left(\xi_{1}+\right)\right)$ problem of $(\mathrm{A} 2)$ is $\lambda=-\xi_{1} \pm \sqrt{-p^{\prime}\left(w\left(\xi_{1}+\right)\right)}$. It is clear that $w\left(\xi_{1}+\right)=w\left(\xi_{2}-\right)$ is a node of (A2). Thus, in order for (A2) to have a solution, it is necessary that $\xi_{1} \geq 0$. On the other hand, however, the same analysis shows that the solvability of (A3) implies $0 \geq \xi_{2}>\xi_{1} \geq 0$. This contradiction proves our assertion.

\section{ACKNOWLEDGMENT}

I would like to thank Professor M. Slemrod for his valuable suggestions and comments as well as continuous support.

I also thank Professor C. M. Dafermos for many discussions and for the opportunity for me to spend some time at the Division of Applied Mathematics at Brown University where this work was done. My thanks also go to Dr. L. Truskinovskii for pointing out one of the mistakes in the Appendix of the previous version of this paper.

This research was supported by U.S. NSF Grant No. DMS-8903083.

\section{REFERENCES}

1. C. M. Dafermos, The entropy rate admissibility criterion for solutions of hyperbolic conservation laws, J. Differential Equations 14 (1973), 202-212.

2. Solution of the Riemann problem for a class of hyperbolic conservation laws by the viscosity method, Arch. Rational Mech. Anal. 52 (1973), 1-9.

3. _ Structure of the solutions of the Riemann problem for hyperbolic conservation laws, Arch. Rational Mech. Anal. 53 (1974), 203-217. 
4. C. M. Dafermos and R. J. DiPerna, The Riemann problem for certain classes of hyperbolic systems of conservation laws, J. Differential Equations 20 (1976), 90-114.

5. C. M. Dafermos, Admissible wave fans in nonlinear hyperbolic systems, Arch. Rational Mech. Anal. 106 (1989), 243-360.

6. Haitao Fan, The structure of the solutions of the gas dynamics equation and the formation of the vacuum state, Quart. Appl. Math. (to appear).

7. H.-T. Fan, A limiting "viscosity" approach to the Riemann problem for the materials exhibiting change of phase. II, Arch. Rational Mech. Anal. (to appear).

8. R. Hagan and M. Slemrod, The viscosity-capillarity criterion for shocks and phase transitions, Arch. Rational Mech. Anal. 83 (1984), 333-361.

9. L. Hsiao, Admissibility criterion and admissible weak solutions of Riemann problem for conservation laws of mixed type, Workshop Proceedings on Nonlinear Evolution Equations that Change Type, IMA Vol. in Math. and its Appl., Springer, Berlin and New York (to appear).

10. __ Uniqueness of admissible solutions of Riemann problem of system of conservation laws of mixed type, J. Differential Equations (to appear).

11. R. D. James, The propagation of phase boundaries in elastic bars, Arch. Rational Mech. Anal. 73 (1980), 125-158.

12. A. S. Kalasnikov, Construction of generalized solutions of quasi-linear equations of first order without convexity conditions as limits of solutions of parabolic equations with a small parameter, Dokl. Akad. Nauk SSSR 127 (1959), 27-30.

13. P. D. Lax, Hyperbolic systems of conservation laws, Comm. Pure Appl. Math. 10 (1957), 537-566.

14. T.-P. Liu, The Riemann problem for general system of conservation laws, J. Differential Equations 18 (1975), 218-234.

15. M. Shearer, Riemann problem for a class of conservation laws of mixed type, J. Differential Equations 46 (1982), 426-443.

16. Nonuniqueness of admissible solutions of Riemann initial value problem for a system of conservation laws of mixed type, Arch. Rational Mech. Anal. 93 (1986), 45-59.

17. _. Dynamic phase transitions in a van der Waals gas, Quart. Appl. Math. 47 (1988), 631-636.

18. M. Slemrod, Admissibility criterion for propagating phase boundaries in a van der Waals fluid, Arch. Rational Mech. Anal. 81 (1983), 301-315.

19. __ Dynamics of first order phase transitions, Phase Transformations and Material Instabilities in Solids (M. Gurtin, ed.), Academic Press, New York, 1984, pp. 163-203.

20. __ A limiting "viscosity" approach to the Riemann problem for materials exhibiting change of phase, Arch. Rational Mech. Anal. 105 (1989), 327-365.

21. M. Slemrod and A. Tzavaras, A limiting viscosity approach for the Riemann problem in isentropic gas dynamics, Indiana Univ. Math. J. (submitted).

22. M. Slemrod, Remarks on the travelling wave theory of the dynamics of phase trasitions, preprint, 1989.

23. V. A. Tupciev, The asymptotic behavior of the solutions of Cauchy problem for the equation $\varepsilon^{2} t u_{x x}=u_{t}+[\phi(u)]_{x}$ that degenerates for $\xi=0$ into the problem of the decay of an arbitrary discontinuity for the case of a rarefaction wave, Zh. Vychisl. Mat. i Mat. Fiz. 12 (1972), 770-775; English transl., U.S.S.R. Comput. Math. and Math. Phys. 12.

24. _ On the method of introducing viscosity in the study of problems involving the decay of discontinuity, Dokl. Akad. Nauk SSSR 211 (1973), 55-58.

Department of Mathematics, University of Wisconsin-Madison, Madison, Wisconsin

Current address: School of Mathematics, Georgia Institute of Technology, Atlanta, Georgia 30332

E-mail address: fan@math.gatech.edu 